\title{
PREDATOR-PREY INTERACTIONS WITH DELAYS DUE TO JUVENILE MATURATION*
}

\author{
KENNETH L. COOKE ${ }^{\dagger}$, RICHARD H. ELDERKIN ${ }^{\dagger}$, AND WENZHANG HUANG ${ }^{\ddagger}$
}

\begin{abstract}
This paper focuses on predator-prey models with juvenile/mature class structure for each of the predator and prey populations in turn, further classified by whether juvenile or mature individuals are active with respect to the predation process. These models include quite general prey recruitment at every stage of analysis, with mass action predation, linear predator mortality as well as delays in the dynamics due to maturation. As a base for comparison we briefly establish that the similar model without delays cannot support sustained oscillation, but it does have predator extinction or global approach to predator-prey coexistence depending on whether the ratio $\alpha$ of per predator predation at prey carrying capacity to the predator death rate is less than or greater than one.

Our first model shows the effect of introducing an invulnerable juvenile prey class, appropriate, e.g., for some host-parasite interactions. In contrast our second model shows the effect of limiting predation to a prey juvenile class. Finally, in a third model we consider an inactive juvenile predator class, which would be appropriate for many traditional situations in which the generation time for the predator is significantly larger than that of the prey. In all cases the introduction of a juvenile class results in a system of three delay-differential equations from which the two equations for the mature class and the nonstructured class can be decoupled. We obtain some global stability results and identify a parameter $\alpha$, similar to the $\alpha$ of the unlagged model, which determines whether or not the predator is driven to extinction. With $\alpha>1$, and considering the maturation age of the juvenile class as a bifurcation parameter, we obtain Hopf bifurcations in our second and third models, while in the case of juvenile prey (in the first model) the unique coexistence equilibrium remains stable for all positive delays. Although the delay is "physically present" in all three models, we obtain scaled, nondimensional replacement models with that physical presence scaled out. After analyzing the scaled equations we show that all our results hold for the original models.

We pursue the bifurcation in the inactive juvenile predator model with numerical simulations. Strikingly similar results over a variety of birth functions are observed. Increases of the maturation delay first produce Hopf bifurcation from steady state to periodic behavior. Even further increase in the delay produces instabilities of the bifurcating periodic solutions with corresponding interesting geometry in a two-dimensional plot of period vs. delay.
\end{abstract}

Key words. predator-prey, host-parasite, age structure, maturation delay, delay differential equations, bifurcation

AMS subject classifications. 92D25, 34K60, 34K18

DOI. $10.1137 / 05063135$

1. Introduction. It is well known that some predators may preferentially attack prey of certain ages or developmental stages. Likewise, the predators themselves may be distinguished in some cases between inactive juveniles and active adults. These situations may be modeled mathematically by dividing the populations into age classes. Our models of this are closely related to those of Hastings ([16], 1983), Murdoch et al. ([22], 1987), the very general ones of Nunney ([25],[26],[27]), and recent work of Gourley and Kuang ([13], 2004). In a model comparable to one of ours (5), Hastings uses a linear mature prey birth rate and a general predation functional

${ }^{*}$ Received by the editors May 11, 2005; accepted for publication (in revised form) August 31, 2005; published electronically March 10, 2006.

http://www.siam.org/journals/siap/66-3/63135.html

$\dagger$ Department of Mathematics, Pomona College, Claremont, CA 91711 (klc04747@pomona.edu, relderkin@pomona.edu).

${ }^{\ddagger}$ Department of Mathematical Sciences, University of Alabama in Huntsville, Huntsville, AL 35899 (huang@ultra.math.uah.edu). This research was supported in part by NSF grant DMS-0204676. 
response to show the possibility of switches between stability and instability of the positive equilibrium as the time delay changes. In contrast we show that this is not possible for our model (5) with its nondecreasing birth rate $N B(N)$. It is known [8] that if $B(N)$ does not satisfy this hypothesis, more complicated dynamic behavior may occur even in a single species population model. In order to analyze models with nonlinear delayed recruitment, we limit our attention to a mass action (i.e., Holling Type I) predation response.

Nunney studied prey-predator systems which are similar to ours, but without the "physical presence" of the delay (that is, occurring outside the arguments of the timevarying states). Assuming the existence of a unique positive equilibrium, he derives a condition for its absolute stability (i.e., linear stability for all delays $R$ ). For our "host-parasite" model (section 3) this condition is satisfied; however, in addition we identify a parameter, $\alpha$, which governs stability of the equilibrium with extinction of the predator. Nunney also considers a similar general system, but in which the delay is due to predator maturation, including our scaled system (50) as special case. Again he assumes existence of a positive equilibrium, and derives a condition for its absolute stability. However, in contrast, with our more specific functional forms we derive conditions on parameters which guarantee Hopf bifurcation to periodic solutions, building on the analysis of Cooke and van den Driessche [7].

Gourley and Kuang consider a model with inactive juvenile predators which is similar to ours in section 5, but with logistic prey recruitment, which we generalize. As in our case, they are faced with the physical presence of the delay in the equations, which causes them (and others) significant difficulty. We exhibit a scaling of the states and parameters which avoids those difficulties (e.g., (8)), yet causes no loss of detail or generality (section 7). Although their predation functional response is initially more general than ours, they specialize, as do we, to the mass-action response before deriving any results. Using our scaling, we are able to analytically produce results, while they relied on computational assistance for theirs. We also implement three examples of birth functions (affine, concave up, and concave down) and conduct numerical experimentation. Over parameter ranges of biological interest the numerical results are very similar across the three function types. However, by extending the maturation delay even further we first find interesting geometries of the bifurcated periodic solutions and at even greater delays find an apparent relation between onset of instabilities and a curious lack of monotonicity in a bifurcation diagram.

We intend our results to be a coherent study across a selection of models that deserve to be considered in the context of each other. We present theoretical results that can be compared across the variation of juvenile/adult roles, both active and inactive, and for both predator and prey. In studying these models, we seek to obtain as much information as we can about how the dynamics of the systems depend on the multiple parameters in the equations, such as attack and mortality rates and the maturation delay. In general, we are looking for conditions that ensure stable equilibrium or bifurcation phenomena. Basic questions are the following: (1) to what extent does the inclusion of the natural mortality parameters alter the qualitative or quantitative behavior of the systems as the maturation delay is varied? (2) is maturation delay more destabilizing in the prey or in the predator? (3) what kinds of destabilizations other than Hopf bifurcations occur as the maturation delay increases? We give partial answers to each of these questions and point the way to further investigation in each case. In general, we find that there is a considerable difference in dynamics depending on whether the prey or predator has a differently behaving 
juvenile class, and on whether the adults or the juveniles are active with respect to the predation process. A moral is that any theoretical or practical study of these situations should carefully take account of the potential for such differences.

We begin the analysis of each of our models by scaling the equations to obtain a dimensionless system in which the delay is no longer physically present. This has the usual advantages of reducing the number of parameters and making the mathematical analysis somewhat simpler and more transparent. Implicit in this strategy is the expectation that the dynamics of the original and scaled systems will be "equivalent" or that the dynamics of one system will be "mirrored" by the dynamics of the other system. Since some of the coefficient parameters of the original system, and some of the transformations in the scaling, depend on the delay, and since we are probing how stability or destabilization depends on the delay, we have included a careful discussion of these and other aspects in section 7 .

The structure of the paper is as follows. In section 2, as a point of departure for what is to come in subsequent sections, we consider a simple predator-prey model that does not include any age structure or resulting delay in recruitment. It consists of two ordinary differential equations in which there is a general prey recruitment function, mass-action predation, and linear mortality in both prey and predator. A simple and natural condition suffices to determine global asymptotic stability of equilibrium; there are no positive periodic solutions.

In section 3 we propose and analyze a model in which the prey has a juvenile class (in addition to an interactive adult class) that is invulnerable to predation while the predator is considered as a single class. For example, this might apply to the sheltered existence of human infants while breast-feeding in a world where parasitism is widespread. The results for our equivalent nondimensionalized system may be stated in terms of a single scaled parameter, $\alpha$, which has a natural interpretation similar to that of $R_{0}$ in many population (especially epidemiological) models. In this case, the system is always dissipative, and when $\alpha>1$ there is a unique equilibrium with both populations present, which must be locally asymptotically stable, independent of the delay. There is no bifurcation to periodic behavior as the delay increases. Later, in section 7 , we discuss what this tells us about the original, nonscaled system.

In section 4 we present a model similar to the one in the preceding section in that there are juvenile and adult prey along with a single class of predators. However, we now assume that survival of juvenile prey is reduced in proportion to the mean population size of predators. Under this hypothesis, our system is now one with "distributed delay." In contrast with the previous case, it turns out that the positive equilibrium is stable for small maturation delays, but unstable with bifurcation to periodic behavior for large ones.

In section 5 we present a model in which there is a single prey population, but a predator with adult and juvenile classes in which the latter do not attack the prey. We show in this case that bifurcation is possible with periodic solutions emerging for large values of the delay under an additional condition on parameters. Here we calculate bifurcation diagrams for examples of the original and the scaled systems, and later in section 7 , we discuss more general relationships.

In section 6 we present results of numerical studies of the bifurcations established in section 5. Before chosing parameter values, we briefly provide interpretations for the more important ones. Then we numerically compare a variety of birth functions in which one is concave down, another concave up, and yet another is affine (over the population range of interest). We arrange that all three resulting models have the same 
interior equilibrium populations and the same populations that are analogous to a carrying capacity (in ODE models), and have the same birth rates at those population levels. We compare the three over a common range of parameters, finding strong similarities. In the case of the affine birth function we extend the Hopf bifurcation branch with increasing delay until a Floquet multiplier leaves the unit disk in the complex plane, signifying onset of instability of the bifurcating periodic solutions. Observing this in a two-dimensional plot of the period of the bifurcating solutions vs. the delay, we see this onset of instability simultaneously as the delay changes from increase to decrease along a backwards S shape just after the period has begun to decrease. At the other end of the backwards $\mathrm{S}$ where the delay begins increasing again, another multiplier leaves the unit disk and the period moves toward increase.

Section 7 addresses the correspondence between the nature of the original models and our analysis of their nondimensional replacements. This analysis is especially motivated by the appearance of the maturation delay physically in the predation coefficients as well as in the populations in the original models, but only within the arguments of the scaled populations in the scaled equations. We show in a precise sense that no bifurcation structure is lost as a result of these scalings.

2. A prototypical model. In order to provide a basis for comparison of our primary results on models with delays due to maturation, we first establish the basic properties exhibited by our model without age structure or corresponding delay. The basic ODE model for a prey population $N$ and a predator population $P$ is

$$
\begin{aligned}
& \frac{d N}{d T}=N B(N)-a N P-d N, \\
& \frac{d P}{d T}=c N P-d_{P} P
\end{aligned}
$$

in which we assume that the per capita prey birth rate $B$ satisfies

$$
\begin{aligned}
B(N) & \geq 0 \quad \text { and } \quad B^{\prime}(N)<0 \quad \text { for } N \geq 0, \\
B(0) & >d>B(\infty) .
\end{aligned}
$$

The assumptions (2a) and (2b) are satisfied, for example, in each of the forms $B(N)=p /(q+N)$ and $B(N)=\exp (-p N)$ where $p$ and $q$ are positive constants (for appropriate $d)$. However see section 3 where the latter form will not work. Furthermore, although (2a) is not satisfied by $B(N)=p-q N$, we adapt this form later to provide a viable example.

It is obvious that (1) always has a trivial equilibrium $(N, P)=(0,0)$ which is an unstable saddle point. Another equilibrium is $\left(N_{0}, 0\right)$, where $N_{0}>0$ is the unique solution of $B(N)=d$, existing by (2). Some simplification of notation can be achieved by scaling $N, P, T$ and various coefficients. In fact, if we set

$$
\begin{aligned}
& x=N / N_{0}, \\
& y=a P / c N_{0}, \\
& t=d_{P} T \\
& \alpha=c N_{0} / d_{P}, \\
& b(x)=B\left(x N_{0}\right) / d_{P}, \quad \gamma=d / d_{P},
\end{aligned}
$$

then the system (1) takes the (nondimensional) form

$$
\begin{aligned}
& \frac{d x}{d t}=x b(x)-\alpha x y-\gamma x, \\
& \frac{d y}{d t}=\alpha x y-y .
\end{aligned}
$$


Notice that in this scaling the transfer from $x$ to $y$ by the scaled predation is perfectly efficient! The properties assumed in (2) take the simpler form

$$
\begin{aligned}
& b(x) \geq 0 \quad \text { and } \quad b^{\prime}(x)<0 \quad \text { for } x \geq 0, \\
& b(0)>\gamma>b(\infty) \quad \text { and } \quad b(1)=\gamma
\end{aligned}
$$

and the equilibria are now at $(0,0)$ and $(1,0)$.

LEMma 1. The system (3) is dissipative, that is, there is a compact set $\Omega$ (in this case of the form $\{(x, y): x, y \geq 0, x+y \leq m\}$ for some $m)$ such that each solution in the first quadrant of the $(x, y)$ plane has a $T_{0}$ such that when $t \geq T_{0},(x(t), y(t)) \in \Omega$.

Proof. With $V(x, y)=x+y$ we have

$$
\dot{V} \stackrel{\text { def }}{=} \frac{d}{d t} V(x(t), y(t))=x b(x)-\gamma x-y .
$$

When $x>1$ this is negative, and when $x \leq 1$ it is negative for sufficiently large $y$. Standard Lyapunov function considerations complete the argument.

THEOREM 2. If $\alpha \leq 1$, then all positive solutions of (3) converge to the equilibrium $(1,0)$ as time $t \rightarrow \infty$.

Proof. The first quadrant of the $(x, y)$ plane is invariant and it follows from the first lemma that every positive solution is bounded. So Poincaré-Bendixson considerations obtain. There is no positive equilibrium; $(0,0)$ is a saddle point and its stable manifold lies on the $y$-axis; and $(1,0)$ is linearly stable. It follows then that $(x(t), y(t)) \rightarrow(1,0)$ as $t \rightarrow \infty$.

It is easily seen that our hypotheses on the birth function $B(x)$ imply the following lemma.

Lemma 3. The system (3) has a positive equilibrium $\left(x^{*}, y^{*}\right)$ if and only if $\alpha>1$, in which case it is unique.

For the remainder of this section we study the case when $\alpha>1$. Since $b^{\prime}\left(x^{*}\right)<$ 0 , it is easy to see that all eigenvalues of the Jacobian matrix $J$ at $\left(x^{*}, y^{*}\right)$ have negative real part, and hence $\left(x^{*}, y^{*}\right)$ is locally asymptotically stable. However, we can establish the stronger result.

THEOREM 4. If $\alpha>1$, then $\left(x^{*}, y^{*}\right)$ is globally asymptotically stable.

Proof. We first establish that any positive nonconstant periodic solution of the system (3) must be asymptotically stable. Suppose that $(\tilde{x}(t), \tilde{y}(t))$ is a positive periodic solution and let $X^{\prime}=A(t) X$ be the linearization of (3) around it. Then a straightforward calculation gives that

$$
A(t)=\left[\begin{array}{cc}
b(\tilde{x}(t))+\tilde{x}(t) b^{\prime}(\tilde{x}(t))-\alpha \tilde{y}(t)-\gamma & -\alpha \tilde{x}(t) \\
\alpha \tilde{y}(t) & \alpha \tilde{x}(t)-1
\end{array}\right] .
$$

Let $T$ be a period of this periodic solution. To establish its asymptotic stability it suffices to show that

$$
\int_{0}^{T} \operatorname{tr} A(t) d t<0
$$

but in fact

$$
\begin{aligned}
\int_{0}^{T} \operatorname{tr} A(t) d t & =\int_{\tilde{x}(0)}^{\tilde{x}(T)} \frac{d x}{x}+\int_{0}^{T} \tilde{x}(t) b^{\prime}(\tilde{x}(t)) d t+\int_{\tilde{y}(0)}^{\tilde{y}(T)} \frac{d y}{y} \\
& =0+\int_{0}^{T} \tilde{x}(t) b^{\prime}(\tilde{x}(t)) d t+0<0
\end{aligned}
$$

since $\tilde{x}(t) b^{\prime}(\tilde{x}(t))<0$ by $(4 \mathrm{a})$. 
Because of this we can now show that the system (3) has no positive periodic solution. Indeed, suppose there were a positive periodic orbit $\Gamma$. The $\alpha$-limits of each point enclosed by $\Gamma$ must be nonempty and also enclosed by $\Gamma$. Since both the positive equilibrium $\left(x^{*}, y^{*}\right)$ and $\Gamma$ are locally asymptotically stable, neither can be contained in an $\alpha$-limit of a point enclosed by $\Gamma$. But there are no other equilibria, and any positive periodic solution must be locally asymptotically stable, so there can be no $\alpha$-limits of points other than $\left(x^{*}, y^{*}\right)$ (the $\alpha$-limit of itself) enclosed by $\Gamma$. This is a contradiction.

Finally, it follows from Lemma 1 that every positive solution is bounded for $t \geq 0$, and therefore must have a nonempty $\omega$-limit. By the preceding argument and Poincaré-Bendixson theory every such limit must contain at least one of the equilibria. However, the two boundary equilibria $(0,0)$ and $(1,0)$ cannot be contained in the necessarily bounded $\omega$-limits of any other points. Hence $\left(x^{*}, y^{*}\right)$ must be in the $\omega$-limit of every interior solution, and since it is locally asymptotically stable it must be the entire limit set.

3. Invulnerable juvenile prey: A host-parasite situation. We now consider the effect of taking into account a juvenile class of prey, which we assume to be invulnerable to predation from birth until an age large enough to warrant inclusion of the class in our model. Taking the model (1) as our starting point, we assume that the juvenile class, consisting of those prey from ages 0 to $R$, is subject to a constant mortality rate $d_{1}$ and so is given by

$$
J(T)=\int_{T-R}^{T} N(s) B(N(s)) e^{-d_{1}(T-s)} d s,
$$

where $N(t)$ is the population of adults of the prey species, and with elementary calculations we find its derivative is

$$
J^{\prime}(T)=N(T) B(N(T))-N(T-R) B(N(T-R)) e^{-d_{1} R}-d_{1} J(T) .
$$

Interpreting the three terms of the last expression, we find that the first is the current rate of juvenile births, while the second is the current rate of maturation of surviving juveniles to adulthood, and the third is current juvenile mortality. These considerations motivate the alteration of our original model to the form

$$
\begin{aligned}
& N^{\prime}(T)=N(T-R) B(N(T-R)) e^{-d_{1} R}-a N(T) P(T)-d N(T), \\
& P^{\prime}(T)=c N(T) P(T)-d_{P} P(T) .
\end{aligned}
$$

We suppose that (2a) continues to hold, as well as the obvious generalization of $(2 b)$,

$$
B(0) e^{-d_{1} R}>d>B(\infty) e^{-d_{1} R} .
$$

In this situation we define $N_{0}$ by the condition

$$
B\left(N_{0}\right) e^{-d_{1} R}=d .
$$

In addition, for $N \geq 0$ we assume that

$$
N B^{\prime}(N)+B(N) \geq 0
$$


or equivalently that the "per capita" recruitment rate, $B(N)$, is kept from decreasing too fast in the sense that $B^{\prime}(N) \geq-B(N) / N$. In contrast with the ODE case, by this assumption we no longer admit the case of $B(N)=\exp (-p N)$. See Cooke et al. [8], where it is shown in a model of a single population that dynamic behavior can be much more complicated when $B(N)$ has the exponential form. Since we want to concentrate on the effects of maturation delays in prey or predator, we have chosen to work with the simpler form. We also wish to point out that many studies in the literature deal with models in which the term $a N P$ is replaced by $\operatorname{Pf}(N)$, where $f$ is called the functional response. We have retained the simpler form $a N P$ in order to isolate the effects of delayed recruitment.

Since (5b, 5c) can be decoupled and solved independently from (5a), we can again restrict our attention to the differential equations (now with delays) for $(N, P)$. We will again find that our analysis is facilitated by a scaling of the variables. If

$$
\begin{array}{ccc}
x=N / N_{0}, & t=d_{P} T, & \alpha=c N_{0} / d_{P}, \\
y=a P / c N_{0}, & r=d_{P} R, & \gamma=d / d_{P}, \\
& b(x)=B\left(x N_{0}\right) e^{-d_{1} R} / d_{P}, &
\end{array}
$$

then the system (5) takes the (nondimensional) form

$$
\begin{aligned}
& \frac{d x}{d t}=x(t-r) b(x(t-r))-\alpha x(t) y(t)-\gamma x(t), \\
& \frac{d y}{d t}=\alpha x(t) y(t)-y(t) .
\end{aligned}
$$

Notice that in this scaling the transfer from $x$ to $y$ by the scaled predation is again perfectly efficient, and the mortality factor, with its physical presence of the delay in $e^{-d_{1} R}$, is scaled out. We will show in section 7 that there is no loss in generality for, e.g., bifurcation as $R$ increases, resulting from this scaling. The properties assumed in (2a), (6), and (7) take the simpler form

$$
\begin{aligned}
b(x) & \geq 0 \quad \text { and } \quad b^{\prime}(x)<0 \quad \text { for } x \geq 0, \\
b(0) & >\gamma>b(\infty) \quad \text { and } \quad b(1)=\gamma, \\
b(x)+x b^{\prime}(x) & \geq 0
\end{aligned}
$$

and the boundary equilibria are now at $(0,0)$ and $(1,0)$.

Lemma 5. The system (9) is dissipative, that is, there is a compact set $\Omega$ (in this case of the form $\{(x, y): x, y \geq 0, x+y \leq m\}$ ) such that each solution in the first quadrant of the $(x, y)$ plane has a $T_{0}$ such that when $t \geq T_{0},(x(t), y(t)) \in \Omega$. Moreover, $\lim \sup _{t \rightarrow \infty} x(t) \leq 1$.

Proof. Since $b(0)>0$ it is easy to check that the first quadrant is forwardly invariant under solutions of (9). With $V(x, y)=x+y$ we have

$$
\dot{V} \stackrel{\text { def }}{=} \frac{d}{d t} V(x(t), y(t))=x(t-r) b(x(t-r))-\gamma x(t)-y(t) .
$$

To set up the analysis of $\dot{V}$, we first establish the boundedness of $x(t)$ for every positive solution.

Let $\bar{x}=\lim \sup _{t \rightarrow \infty} x(t)$. Suppose, contrary to our claim, that $\bar{x}>1$. Since the case in which $\bar{x}$ is infinite is similar to the finite case, but slightly less complicated, we will consider only the finite case. Then we can find a sequence $\left\{t_{n}\right\}$ with $t_{n} \rightarrow \infty$ 
as $n \rightarrow \infty$ such that $x\left(t_{n}\right) \rightarrow \bar{x}, x^{\prime}\left(t_{n}\right) \rightarrow 0$, and $\lim \sup _{n \rightarrow \infty} x\left(t_{n}-r\right) \leq \bar{x}$. Then we have

$$
\begin{aligned}
\lim _{n \rightarrow \infty} x^{\prime}\left(t_{n}\right) & \leq \lim _{n \rightarrow \infty} \sup \left(x\left(t_{n}-r\right) b\left(x\left(t_{n}-r\right)\right)-\gamma x\left(t_{n}\right)\right) \\
& =\lim _{n \rightarrow \infty} \sup _{n \rightarrow \infty}\left(x\left(t_{n}-r\right) b\left(x\left(t_{n}-r\right)\right)\right)-\gamma \bar{x} \\
& \leq(b(\bar{x})-\gamma) \bar{x}<0,
\end{aligned}
$$

contradicting our choice of $\left\{t_{n}\right\}$ so that $x^{\prime}\left(t_{n}\right) \rightarrow 0$. Because of this every $x(t)$ has $\lim \sup _{t \rightarrow \infty} x(t) \leq 1$.

Finally we want to show that $V(x(t), y(t))$ is decreasing whenever it has sufficiently large values. To this end, let $\tilde{x}>1$ and then choose $\tilde{y}$ such that $x b(x)-y<0$ when $x \leq \tilde{x}$ and $y \geq \tilde{y}$. Now when $V(x(t), y(t))>\tilde{x}+\tilde{y}$ for large $t$, we must have $x(t), x(t-r) \leq \tilde{x}$ (by the previous paragraph) and hence $y(t)>\tilde{y}$ so that $\dot{V}<x(t-r) b(x(t-r))-y(t)<\tilde{x} b(\tilde{x})-\tilde{y}<0$. Thus for any solution after sufficiently long time, we have $\dot{V}<0$ whenever $V(x(t), y(t))>\tilde{x}+\tilde{y}$. Standard Lyapunov function considerations complete the argument.

Corollary 6. Suppose $\alpha<1$. Then all positive solutions of (9) converge to $(1,0)$ as $t \rightarrow \infty$.

Proof. First we establish that $y(t) \rightarrow 0$ as $t \rightarrow \infty$. Let $\beta>0$ such that $\alpha<\beta<1$, and let $(x(t), y(t))$ be a positive solution of (9). By Lemma 5 there is a sufficiently large $t^{*}$ such that $\alpha x(t) \leq \beta$ for all $t \geq t^{*}$. This implies that

$$
y^{\prime}(t) \leq-(1-\beta) y(t), \quad t \geq t^{*} .
$$

Therefore for $t \geq t^{*}$ we have

$$
0 \leq y(t) \leq y\left(t^{*}\right) e^{-(1-\beta)\left(t-t^{*}\right)} \rightarrow 0 \quad \text { as } t \rightarrow \infty .
$$

It follows that $y(t) \rightarrow 0$ as $t \rightarrow \infty$.

Next we claim that

$$
M=\lim _{t \rightarrow \infty} \inf x(t)>0 .
$$

Suppose to the contrary that $M=0$. Then since $x(t)>0$ for all $t \geq 0$, one is able to choose a sequence $\left\{t_{n}\right\}$ having the properties that

$$
\begin{aligned}
x\left(t_{n}-r\right) & \geq x\left(t_{n}\right), \\
x^{\prime}\left(t_{n}\right) & \leq 0
\end{aligned}
$$

for all $n \geq 1$ and

$$
\lim _{n \rightarrow \infty} x\left(t_{n}\right)=\lim _{n \rightarrow \infty} x^{\prime}\left(t_{n}\right)=0 .
$$

Since $\lim _{n \rightarrow \infty} x\left(t_{n}\right)=0$, for which there is a $J$ such that

$$
x\left(t_{n}\right)<\frac{1}{2}, \quad n \geq J,
$$

and since $b(x)$ is strictly decreasing, there is an $\varepsilon>0$ such that

$$
b\left(x\left(t_{n}\right)\right) \geq b(1)+\varepsilon=\gamma+\varepsilon, \quad n \geq J .
$$


Moreover since $y(t) \rightarrow 0$ as $t \rightarrow \infty$, there exists $J_{1} \geq J$ such that

$$
\alpha y\left(t_{n}\right)<\varepsilon, \quad n \geq J_{1} .
$$

Then for $n \geq J_{1}$ we have from (11), the increasing of $x b(x)$ and (11), and (12-14) that

$$
\begin{aligned}
0 & \geq x^{\prime}\left(t_{n}\right) \\
& =x\left(t_{n}-r\right) b\left(x\left(t_{n}-r\right)\right)-\left[\alpha y\left(t_{n}\right)+\gamma\right] x\left(t_{n}\right) \\
& \geq x\left(t_{n}\right) b\left(x\left(t_{n}\right)\right)-\left[\alpha y\left(t_{n}\right)+\gamma\right] x\left(t_{n}\right) \\
& >x\left(t_{n}\right)(\gamma+\varepsilon)-\left[\alpha y\left(t_{n}\right)+\gamma\right] x\left(t_{n}\right) \\
& >0 .
\end{aligned}
$$

This contradiction establishes that $M=\lim _{t \rightarrow \infty} \inf x(t)>0$.

Finally we can show that $M=1$. Let $t_{n} \rightarrow \infty$ in such a way that $x\left(t_{n}\right) \rightarrow M$ as $n \rightarrow \infty$ and

$$
\lim _{n \rightarrow \infty} x^{\prime}\left(t_{n}\right)=0 .
$$

Since $\left\{x\left(t_{n}-r\right)\right\}$ is a bounded sequence, without loss of generality (or by choosing a subsequence) we can suppose that $x\left(t_{n}-r\right)$ converges,

$$
\lim _{n \rightarrow \infty} x\left(t_{n}-r\right)=M_{1} .
$$

By Lemma 5 and the definition of $M$ it is obvious that

$$
M \leq M_{1} \leq 1 .
$$

Therefore $(15,16)$ yield

$$
\begin{aligned}
0 & =\lim _{n \rightarrow \infty} x^{\prime}\left(t_{n}\right) \\
& =\lim _{n \rightarrow \infty} x\left(t_{n}-r\right) b\left(x\left(t_{n}-r\right)\right)-\gamma \lim _{n \rightarrow \infty} x\left(t_{n}\right) \\
& =M_{1} b\left(M_{1}\right)-\gamma M .
\end{aligned}
$$

We now show that this equality forces $M=M_{1}=1$. For if $M<1$, then either $M<M_{1}$ or $M=M_{1}<1$. If $M<M_{1}$, then we have

$$
M_{1} b\left(M_{1}\right) \geq M_{1} b(1)=M_{1} \gamma>\gamma M,
$$

a contradiction of the established equality. On the other hand, if $M=M_{1}<1$, then

$$
M_{1} b\left(M_{1}\right)>M_{1} b(1)=M_{1} \gamma=\gamma M,
$$

again contradicting the equality.

Hence $M=\liminf _{t \rightarrow \infty} x(t)=1$, which, in conjunction with Lemma 5 gives $x(t) \rightarrow 1$.

Now let us suppose $\alpha>1$ (i.e., $c N_{0}>d_{P}$ ). Then the system (9) has a unique positive equilibrium $\left(x^{*}, y^{*}\right)$. We proceed to study the local stability of this positive equilibrium. 
First, following a straightforward calculation one is able to verify that the linearization of (9) at the positive equilibrium takes the form

$$
\begin{aligned}
& u^{\prime}(t)=\left[x^{*} b^{\prime}\left(x^{*}\right)+b\left(x^{*}\right)\right] u(t-r)-\left(\alpha y^{*}+\gamma\right) u(t)-\alpha x^{*} v(t), \\
& v^{\prime}(t)=\alpha y^{*} u(t)
\end{aligned}
$$

TheOREM 7. The interior equilibrium $\left(x^{*}, y^{*}\right)$ (which exists if and only if $\alpha>1$ ) is locally asymptotically stable for all $r \geq 0$.

Proof. Let $K=\left[x^{*} b^{\prime}\left(x^{*}\right)+b\left(x^{*}\right)\right]$. The characteristic equation of (17) is given by

$$
\begin{aligned}
\Delta(\lambda, r) & =\operatorname{det}\left[\begin{array}{cc}
K e^{-\lambda r}-\lambda-\left(\alpha y^{*}+\gamma\right) & -\alpha x^{*} \\
\alpha y^{*} & -\lambda
\end{array}\right] \\
& =\lambda^{2}-\lambda K e^{-\lambda r}+\lambda\left(\alpha y^{*}+\gamma\right)+\alpha^{2} x^{*} y^{*} .
\end{aligned}
$$

Note that $b^{\prime}\left(x^{*}\right)<0$ and $b\left(x^{*}\right)=\alpha y^{*}+\gamma$. Hence from the assumption (10c) we have

$$
\alpha y^{*}+\gamma>|K|
$$

We observe that

$$
\Delta(\lambda, 0)=\lambda^{2}+\left(\alpha y^{*}+\gamma-K\right) \lambda+\alpha^{2} x^{*} y^{*} .
$$

It follows from (18) that all coefficients of $\Delta(\lambda, 0)$ are positive, and hence all zeros of $\Delta(\lambda, 0)$ have the negative real parts. We claim that for each fixed $r>0$, all zeros of $\Delta(\lambda, r)$ are located in the left half complex plane. If this is not true, then there must be a $r>0$ and $v \in \Re$ such that $\Delta(i v, r)=0$, or equivalently,

$$
\Delta(i v, r)=-v^{2}-i v K e^{-i \nu r}+i v\left(\alpha y^{*}+\gamma\right)+\alpha^{2} x^{*} y^{*}=0 .
$$

That is,

$$
-v^{2}+\alpha^{2} x^{*} y^{*}+i v\left(\alpha y^{*}+\gamma\right)=i v K e^{-i v r}
$$

This yields that

$$
\left|-v^{2}+\alpha^{2} x^{*} y^{*}+i v\left(\alpha y^{*}+\gamma\right)\right|^{2}=\left|i v K e^{-i v r}\right|^{2} .
$$

Hence we obtain that

$$
\left(\alpha^{2} x^{*} y^{*}-v^{2}\right)^{2}+\left(\alpha y^{*}+\gamma\right)^{2} v^{2}=K^{2} v^{2} .
$$

However, since $\left(\alpha y^{*}+\gamma\right)^{2}>K^{2}$, the above equality can never hold for $v \in \Re$. This contradiction establishes our result.

4. Invulnerable mature prey. Next we consider a case similar to that in the previous section in distinguishing between juvenile and adult prey, but opposite from it in assuming that predation affects juvenile prey but not mature prey. As before, we begin with the idea that the class of juveniles at time $T$ consists of all those prey surviving from birth in the time interval $[T-R, T]$ and write

$$
J(T)=\int_{T-R}^{T} N(s) B(N(s)) e^{-M} d s,
$$


where $N$ and $B$ respectively represent the mature prey class and its per capita birth rate and $M$ represents mortality effects. In addition to the "natural" mortality given by a constant per capita rate $d_{1}$ and represented in the previous section by $-d_{1}(T-s)$, we now include mortality by predation that is jointly proportional to the length of time $(T-s)$ such predation can occur and the average size of the predator class (denoted by $P$ as before) over the interval $[s, T]$, so that

$$
M=d_{1}(T-s)+d_{2}(T-s)\left(\frac{1}{T-s} \int_{s}^{T} P(u) d u\right)=\int_{s}^{T}\left[d_{1}+d_{2} P(u)\right] d u
$$

and hence

$$
J(T)=\int_{T-R}^{T} N(s) B(N(s)) e^{-\int_{s}^{T}\left[d_{1}+d_{2} P(u)\right] d u} d s .
$$

Then from elementary calculations we find that

$$
\begin{aligned}
\frac{d J}{d T}(T)= & N(T) B(N(T))-N(T-R) B(N(T-R)) e^{-\int_{T-R}^{T}\left[d_{1}+d_{2} P(u)\right] d u} \\
& -\left[d_{1}+d_{2} P(T)\right] J(T) .
\end{aligned}
$$

The three terms comprising $d J / d T$ have nice interpretations: the first as current births, the second as loss to maturation of those who survived from birth (at time $T-R$ ) to the present, and the third as current loss due to the combination of constant per capita mortality and "mass-action" predation.

Assuming no predation directly on mature prey we arrive at the system of equations for $(J, N, P)$ (with notation similar to that in the previous section)

$$
\begin{aligned}
& \frac{d N}{d T}(T)=N(T-R) B(N(T-R)) F\left(\int_{T-R}^{T} P(u) d u\right)-d N(T), \\
& \frac{d P}{d T}(T)=k P(T) J(T)-d_{P} P(T),
\end{aligned}
$$

wherein we assume about $F$ only the conditions

$$
1 \geq F(0)>0, \quad F(\infty)=0, \quad F^{\prime}(Z)<0, \quad Z>0
$$

as motivated by our considerations in (19) where $F(Z)=e^{-d_{1} R-d_{2} Z}$. Notice that if the juvenile prey $J(T)$ were a constant proportional part of the mature prey population then the last two equations would decouple from the first and could be solved independently. Thus, with the goal of facilitating comparison with our two other models, we make the a priori assumption that $J(T)=C N(T)$. Thus, we consider the system

$$
\begin{aligned}
& \frac{d N}{d T}(T)=N(T-R) B(N(T-R)) F\left(\int_{T-R}^{T} P(u) d u\right)-d N(T) \\
& \frac{d P}{d T}(T)=c P(T) N(T)-d_{P} P(T) .
\end{aligned}
$$

In order to achieve a satisfactory scaling of the model we desire an $N_{0}$ that functions like a prey carrying capacity in the absence of predators. As before we suppose 
that the conditions (2a) and (7) on $B$ continue to hold, as well as the modification of (2b) given by

$$
B(0) F(0)>d>B(\infty) F(0) .
$$

In this situation, $N_{0}$ is uniquely determined by the condition

$$
B\left(N_{0}\right) F(0)=d,
$$

providing an equilibrium at $\left(N_{0}, 0\right)$. Under the scaling

$$
\begin{gathered}
x=N / N_{0}, \quad t=d_{P} T, \quad \alpha=c N_{0} / d_{P}, \\
y=P / N_{0}, \quad r=d_{P} R, \quad \gamma=d / d_{P}, \\
b(x)=B\left(x N_{0}\right) / d_{P}, \quad f(z)=F\left(\frac{N_{0}}{d_{P}} z\right)
\end{gathered}
$$

we find that (20) becomes the dimensionless system

$$
\begin{aligned}
& \frac{d x}{d t}(t)=x(t-r) b(x(t-r)) f\left(\int_{-r}^{0} y(t+s) d s\right)-\gamma x(t), \\
& \frac{d y}{d t}(t)=\alpha x(t) y(t)-y(t) .
\end{aligned}
$$

Notice that time has again been scaled by the reciprocal of the predator death rate and that $b(\cdot)$ and $f(\cdot)$ satisfy conditions similar to (10) in section 3 :

$$
\begin{array}{rlrl}
b(x) & \geq 0 \quad \text { and } \quad b^{\prime}(x)<0 \quad & \text { for } x \geq 0, \\
b(0) f(0) & >\gamma>b(\infty) f(0) \quad \text { and } \quad & & b(1) f(0)=\gamma, \\
b(x)+x b^{\prime}(x) & \geq 0 & & \\
1 & \geq f(0)>0, \quad f(\infty)=0, \quad \frac{d f}{d z}<0, \quad z>0 .
\end{array}
$$

Just as in the systems (3) and (9), this system has no strictly positive equilibrium if $\alpha<1$ and has a unique positive equilibrium if $\alpha>1$.

THEOREM 8. If $\alpha<1$, then all positive solutions of (22) converge to the equilibrium $(1,0)$.

Proof. Following essentially the same argument as in the second paragraph of the proof of Lemma 5 , one establishes that $\limsup _{t \rightarrow \infty} x(t) \leq 1$. Given a positive solution $(x(t), y(t))$ we can find a $T$ such that for $t \geq T, \alpha x(t)-1$ is negative and bounded away from zero. Since

$$
y^{\prime}(t) \leq(\alpha x(t)-1) y(t),
$$

we find that $y(t) \rightarrow 0$ as claimed. The rest of the argument is similar to that given for proof of Corollary 6 .

For the rest of this section we consider the remaining case in which $\alpha>1$. In this case an equilibrium $\left(x^{*}, y^{*}\right)$ must satisfy $x^{*}=1 / \alpha<1$ so that

$$
b\left(x^{*}\right) f\left(z^{*}\right)-\gamma=0
$$

has a unique solution $z^{*}=r y^{*}>0$. Thus for each fixed $r>0,(22)$ has the unique positive equilibrium $\left(x^{*}, y^{*}\right)=\left(1 / \alpha, z^{*} / r\right)$. We will show that an increase of the time 
delay $r$ will destabilize the stability of this positive equilibrium and cause a Hopf bifurcation.

First a direct computation yields that the linearization of (22) around the positive equilibrium is given by

$$
\begin{aligned}
& u^{\prime}(t)=a u(t-r)-\gamma u(t)-b \int_{-r}^{0} v(t+s) d s, \\
& v^{\prime}(t)=\alpha y^{*} u(t)
\end{aligned}
$$

where

$$
\begin{aligned}
a & =\left[b\left(x^{*}\right)+x^{*} b^{\prime}\left(x^{*}\right)\right] f\left(z^{*}\right), \\
b & =-x^{*} b\left(x^{*}\right) \frac{d f}{d z}\left(z^{*}\right)>0 .
\end{aligned}
$$

Therefore the characteristic equation is given by

$$
\begin{aligned}
\Delta(\lambda, r) & =\operatorname{det}\left[\begin{array}{cc}
a e^{-\lambda r}-\gamma-\lambda & -b \int_{-r}^{0} e^{\lambda s} d s \\
\alpha y^{*} & -\lambda
\end{array}\right] \\
& =\lambda^{2}+\gamma \lambda-a \lambda e^{-\lambda r}+\frac{\beta\left(1-e^{-\lambda r}\right)}{r \lambda} \\
& =0
\end{aligned}
$$

where $\beta=\alpha b z^{*}$.

To study the location of eigenvalues of the characteristic equation (28), an important first step is to investigate the existence of eigenvalues that lie on the imaginary axis of the complex plane and the direction in which these eigenvalues cross that axis as the delay increases. We note that if $\Delta(i v, r)=0$, then $\Delta(-i v, r)=0$ so that we shall only search for eigenvalues $i v$ with $v>0$. Letting

$$
h(\lambda, r)=r \lambda^{3}+\gamma r \lambda^{2}-a r \lambda^{2} e^{-\lambda r}+\beta\left(1-e^{-\lambda r}\right),
$$

it is clear that $\Delta(\lambda, r)=0$ if and only if $h(\lambda, r)=0$ for $\lambda \neq 0$ and $r>0$.

LEMma 9. There are infinitely many positive pairs of $(i v, r)$ with $r>0$ and $v=v(r)>0$ such that $\Delta(i v, r)=0$. However, there is an interval $0<r<r_{1}$ such that $\Delta(\lambda, r)$ has no purely imaginary zeros.

Proof. Note that with $r>0, \gamma=b\left(x^{*}\right) f\left(z^{*}\right)$, the assumptions on $b$ and $f$ imply that

$$
\gamma>|a|, \quad \beta>0
$$

Notice that in $\Delta(\lambda, r)$, neither $a$ nor $\beta$ depends on $r$. Thus

$$
\Delta(\lambda, 0)=\lambda^{2}+(\gamma-a) \lambda+\beta
$$

and the interval $\left(0, r_{1}\right)$ on which there are no purely imaginary characteristic zeros follows immediately from continuity.

Considering $\lambda=i v$, if

$$
h(i v, r)=0
$$


for some $v>0$ and $r>0$, then we have

$$
\frac{-i r v^{3}+\beta-\gamma r v^{2}}{\left(\beta-a r v^{2}\right)}=e^{-i v r}
$$

so that

$$
\left|-i r v^{3}+\beta-\gamma r v^{2}\right|^{2}=\left|\beta-a r v^{2}\right|^{2},
$$

or

$$
r^{2} v^{6}+\left(\beta-\gamma r v^{2}\right)^{2}=\left(\beta-a r v^{2}\right)^{2},
$$

and finally

$$
r v^{4}+r\left(\gamma^{2}-a^{2}\right) v^{2}=2 \beta(\gamma-a) .
$$

We can solve (35) uniquely for $v^{2}=v^{2}(r)$,

$$
v^{2}=\frac{1}{2}\left(-\left(\gamma^{2}-a^{2}\right)+\sqrt{\left(\gamma^{2}-a^{2}\right)^{2}+\frac{8 \beta(\gamma-a)}{r}}\right) .
$$

Thus we have shown that if $h(i v, r)=0$, then $v=v(r)$ with $v>0$ must satisfy (36). However, it is really the converse question that we must answer: if $v(r)$ is the positive branch of (36), do we have $h(i v(r), r)=0$ ? However, for $v>0,(36)$ is equivalent to (33), so that if we let $W(r)$ denote the left-hand side of (32) with $v=v(r)$, then $|W(r)|=1$ for all $r>0$.

Multiplying (36) by $r^{2}$ we have

$$
(r v)^{2}=\frac{1}{2}\left(-r^{2}\left(\gamma^{2}-a^{2}\right)+\sqrt{r^{4}\left(\gamma^{2}-a^{2}\right)^{2}+8 r^{3} \beta(\gamma-a)}\right) .
$$

From this, one immediately sees that

$$
\lim _{r \rightarrow 0}[r v(r)]^{2}=0 .
$$

Moreover, rationalizing the numerator in (37) we have

$$
(r v)^{2}=\frac{8 r^{3} \beta(\gamma-a)}{2\left(r^{2}\left(\gamma^{2}-a^{2}\right)+\sqrt{r^{4}\left(\gamma^{2}-a^{2}\right)^{2}+8 r^{3} \beta(\gamma-a)}\right)}
$$

which yields

$$
\lim _{r \rightarrow \infty}[r v(r)]^{2}=\infty .
$$

It follows from (38) and (39) that

$$
\lim _{r \rightarrow 0} r v(r)=0 \quad \text { and } \quad \lim _{r \rightarrow \infty} r v(r)=\infty .
$$

Next from (34) we have that $\beta-\operatorname{rav}^{2}(r)>0$ for all $r>0$. For if this inequality does not hold for some $r>0$, then we have $\left|\beta-a r v^{2}(r)\right|=a r v^{2}(r)-\beta$. Since $\gamma>a$ we have $\gamma r v^{2}(r)-\beta>a r v^{2}(r)-\beta$. It would therefore follow that

$$
r^{2} v^{6}(r)+\left(\beta-\gamma r v^{2}(r)\right)^{2}>\left(\beta-a r v^{2}(r)\right)^{2},
$$

contradicting (34). 
Finally, substitute $v=v(r)$ into (32). Letting $r$ increase from 0 to $\infty$ and using (40), we find that $e^{-i r v}$ on the right side traces out the unit circle infinitely often, while on the left side $W(r)$ remains in the lower half of the unit circle. Now one easily sees that there are infinitely many positive $r$ 's such that $h(i v(r), r)$ $=0$.

LEMma 10. Let $\lambda(r)$ be a branch of zeros of $h(\lambda, r)$ defined on an interval I such that for some $0<r_{0} \in I, \lambda\left(r_{0}\right)=i v_{0}$ with $v_{0}>0$. Then $v_{0}$ is a simple zero and

$$
\operatorname{Re}\left(\frac{d \lambda\left(r_{0}\right)}{d r}\right)>0
$$

Proof. Since $h(\lambda(r), r) \equiv 0$, we have

$$
\frac{\partial h(\lambda, r)}{\partial \lambda} \frac{d \lambda}{d r}=-\frac{\partial h(\lambda, r)}{\partial r} .
$$

Hence

$$
\overline{\left[\frac{\partial h(\lambda, r)}{\partial \lambda}\right]} \frac{\partial h(\lambda, r)}{\partial \lambda} \frac{d \lambda}{d r}=-\overline{\left[\frac{\partial h(\lambda, r)}{\partial \lambda}\right]} \frac{\partial h(\lambda, r)}{\partial r},
$$

where $\bar{z}$ denotes the conjugate of a complex number $z$. It follows from this equality that

$$
\operatorname{sign}\left(\operatorname{Re} \frac{d \lambda\left(r_{0}\right)}{d r}\right)=\operatorname{sign}\left(\operatorname{Re}\left\{-\overline{\left[\frac{\partial h\left(i v_{0}, r_{0}\right)}{\partial \lambda}\right]} \frac{\partial h\left(i v_{0}, r_{0}\right)}{\partial r}\right\}\right) .
$$

For notational simplicity from now on we use $v$ and $r$ instead of $v_{0}$ and $r_{0}$. First from (29) we deduce that

$$
i v^{3}+\gamma v^{2}-a v^{2} e^{-i v r}=\frac{\beta}{r}\left(1-e^{-i v r}\right),
$$

or equivalently, after complex conjugation

$$
v^{2}+i \gamma v-i a v e^{i v r}=\frac{i \beta}{r v}\left(1-e^{i v r}\right) .
$$

Following a straightforward computation and with the use of (41) and (42) we have

$$
\begin{gathered}
\overline{\left[\frac{\partial h(i v, r)}{\partial \lambda}\right]}=r\left[-3 v^{2}-i 2 \gamma v+i 2 a v e^{i v r}+\left(\beta-a r v^{2}\right) e^{i v r}\right] \\
=r\left[-3\left(v^{2}+i \gamma v-i a v e^{i v r}\right)+i(\gamma-a) v+i a v\left(1-e^{i v r}\right)\right] \\
+r\left(\beta-a r v^{2}\right) e^{i v r} \\
=r\left[i(\gamma-a) v+i\left(a v-\frac{3 \beta}{r v}\right)\left(1-e^{i v r}\right)+\left(\beta-a r v^{2}\right) e^{i v r}\right] \\
-\frac{\partial h(i v, r)}{\partial r}=i v^{3}+\gamma v^{2}-a v^{2} e^{-i v r}-i v\left(\beta-a r v^{2}\right) e^{-i v r} \\
=\frac{\beta}{r}\left(1-e^{-i v r}\right)-i v\left(\beta-a r v^{2}\right) e^{-i v r} .
\end{gathered}
$$


Let

$$
\Phi=-\overline{\left[\frac{\partial h(i v, r)}{\partial \lambda}\right]} \frac{\partial h(i v, r)}{\partial r} .
$$

It is clear that if $\operatorname{Re} \Phi>0$, then $\frac{\partial h(i v, r)}{\partial \lambda} \neq 0$ which will imply that $i v=i v_{0}$ is a simple eigenvalue and $\operatorname{Re} \frac{d \lambda\left(r_{0}\right)}{d r}>0$. Hence to complete the proof of Lemma 10, it is sufficient to show that $\operatorname{Re} \Phi>0$. First by (43) and (44) we have

$$
\begin{aligned}
\operatorname{Re} \Phi= & \operatorname{Re}\left\{\beta\left(1-e^{-i v r}\right)\left[i(\gamma-a) v+\left(\beta-a r v^{2}\right) e^{i v r}\right]\right\} \\
& +\operatorname{Re}\left\{-i v r\left(\beta-a r v^{2}\right) e^{-i v r}\left[-3 v^{2}-i 2 \gamma v+i 2 a v e^{i v r}\right]\right\} \\
= & \operatorname{Re} \Phi_{1}+\operatorname{Re} \Phi_{2}
\end{aligned}
$$

with

$$
\begin{aligned}
& \Phi_{1}=\beta\left(1-e^{-i v r}\right)\left[i(\gamma-a) v+\left(\beta-a r v^{2}\right) e^{i v r}\right] \\
& \Phi_{2}=-i v r\left(\beta-a r v^{2}\right) e^{-i v r}\left[-3 v^{2}-i 2 \gamma v+i 2 a v e^{i v r}\right] .
\end{aligned}
$$

Following a further calculation we have

$$
\begin{aligned}
& \operatorname{Re} \Phi_{1}=-\beta(\gamma-a) v \sin (v r)-\beta\left(\beta-a r v^{2}\right)[1-\cos (v r)], \\
& \operatorname{Re} \Phi_{2}=\left(\beta-a r v^{2}\right)\left[3 r v^{3} \sin (v r)-2 \gamma r v^{2} \cos (v r)+2 a r v^{2}\right] .
\end{aligned}
$$

Next, by separating the real and imaginary parts of (32) we obtain

$$
\begin{aligned}
& \left(\beta-a r v^{2}\right) \cos (v r)=\beta-\gamma r v^{2}, \\
& \left(\beta-a r v^{2}\right) \sin (v r)=r v^{3} .
\end{aligned}
$$

With the use of (35), (45), and (46) we finally arrive at

$$
\begin{aligned}
\operatorname{Re} \Phi= & -\beta(\gamma-a) v \sin (v r)-\beta\left(\beta-a r v^{2}\right)+\beta\left(\beta-\gamma r v^{2}\right) \\
& +3 r^{2} v^{6}-2 \gamma r v^{2}\left(\beta-\gamma r v^{2}\right)+2 a r v^{2}\left(\beta-a r v^{2}\right) \\
= & -\beta(\gamma-a) v \sin (v r)-3 r \beta v^{2}(\gamma-a)+3 r^{2} v^{6}+2 r^{2} v^{4}\left(\gamma^{2}-a^{2}\right) \\
= & -\beta(\gamma-a) v \sin (v r)+r^{2} v^{6}+r \beta v^{2}(\gamma-a) \\
& +2 r^{2} v^{6}+2 r^{2} v^{4}\left(\gamma^{2}-a^{2}\right)-4 r v^{2} \beta(\gamma-a) \\
= & -\beta(\gamma-a) v \sin (v r)+r^{2} v^{6}+r \beta v^{2}(\gamma-a) \\
> & \beta(\gamma-a) v[r v-\sin (v r)] \\
\geq & 0 . \quad \square
\end{aligned}
$$

Now we are ready to prove the following result.

Theorem 11. Suppose that $\alpha>1$. Then there is a sequence $\left\{r_{n}\right\}_{n=1}^{\infty}$ with

$$
0<r_{1}<r_{2}<\cdots<r_{n}<\cdots
$$

such that the following hold:

1. If $0<r<r_{1}$, then the positive equilibrium $\left(x^{*}, y^{*}\right)=\left(1 / \alpha, z^{*} / r\right)$ of (22) is linearly stable. If $r>r_{1}$ then that positive equilibrium is linearly unstable.

2. For each $n \in \mathbf{N}$ there is a $v_{n}>0$ such that $\lambda= \pm i v_{n}$ are eigenvalues associated with the equilibrium $\left(x^{*}, y^{*}\right)$ of $(22)$ at $r=r_{n}$ and there is a Hopf bifurcation there. 
Proof. Using Lemmas 9 and 10 with the application of classical results on stability switches (for example, see [7]) and on Hopf bifurcation (e.g., [10], p. 291), we need only to show that all solutions of the characteristic equation

$$
\Delta(\lambda, r)=\lambda^{2}+\gamma \lambda-a \lambda e^{-\lambda r}+\frac{\beta\left(1-e^{-\lambda r}\right)}{r \lambda}=0
$$

have negative real parts for all $r \in\left[0, r_{1}\right)$, where $r_{1}$ is taken to be the smallest positive delay at which the characteristic function has a purely imaginary zero. The existence of $r_{1}$ is guaranteed by Lemma 9 .

Now for a characteristic root $\lambda=\lambda(r)=u+i v$ with $r \geq 0$ and $u \geq 0$ we have

$$
\begin{aligned}
\left|1-e^{-\lambda r}\right| & \leq\left|1-e^{-u r}+e^{-u r}(1-\cos (v r)+i \sin (v r))\right| \\
& \leq\left|1-e^{-u r}\right|+|1-\cos (v r)|+|\sin (v r)| \\
& \leq|u r|+2|v r| \leq 3|\lambda r| .
\end{aligned}
$$

From this we can conclude that

$$
\left|\frac{\beta\left(1-e^{-\lambda r}\right)}{r \lambda}\right| \leq 3 \beta .
$$

It now follows from this that there is a sufficiently large constant $C$ such that for all $\lambda \in \mathbf{C}$ with $\operatorname{Re}(\lambda) \geq 0$ and $|\lambda| \geq C$,

$$
\lambda^{2}+\gamma \lambda-a \lambda e^{-\lambda r}+\frac{\beta\left(1-e^{-\lambda r}\right)}{r \lambda} \neq 0 .
$$

Since $\Delta(\lambda, r)$, with $\Delta(\lambda, 0)$ as in (30), is analytic in $\lambda \neq 0$ and continuous in $r \geq 0$, it follows that its number of zeros on $\operatorname{Re} \lambda \geq 0$ is constant for $r \in\left[0, r_{1}\right)$. Since $\Delta(\lambda, 0)$ has only zeroes with negative real part, our result holds.

5. Inactive juvenile predators. We now consider the effect of taking into account an inactive juvenile class of predator. Thus all predation is done by the adult predators which we still denote by $P$, but we change $J$ to denote the juvenile predators and keep $N$ to denote the prey. Taking the model (1) again as our starting point, we assume that the juvenile class (consisting of those predators from ages 0 to $R$ ) is the direct beneficiary of predation and is subject to a constant mortality rate $d_{1}$ and so is given by

$$
J(T)=\int_{T-R}^{T} c N(s) P(s) e^{-d_{1}(T-s)} d s
$$

with derivative

$$
J^{\prime}(T)=c N(T) P(T)-c N(T-R) P(T-R) e^{-d_{1} R}-d_{1} J(T) .
$$

Interpreting the three terms of the last expression, we find that the first is the current rate of juvenile births, while the second is the current rate of maturation of surviving juveniles to adulthood, and the third is current juvenile mortality. Thus

$$
\begin{aligned}
& N^{\prime}(T)=N(T) B(N(T))-a N(T) P(T)-d N(T) \\
& P^{\prime}(T)=c N(T-R) P(T-R) e^{-d_{1} R}-d_{P} P(T) .
\end{aligned}
$$


We suppose that the conditions (2a), (2b), and (7) on $B$ continue to hold. As in section 2 we define $N_{0}$ by the condition

$$
B\left(N_{0}\right)=d .
$$

Since (48a) and (48b) can be decoupled and solved independently from (47), we can again restrict our attention to the differential equations (with delays) for $(N, P)$.

We will again find that our analysis is facilitated by a scaling of the variables. If

$$
\begin{array}{ccc}
x=N / N_{0}, & t=d_{P} T, & \alpha=c e^{-d_{1} R} N_{0} / d_{P}, \\
y=a e^{d_{1} R} P / c N_{0}, & r=d_{P} R, & \gamma=d / d_{P},
\end{array}
$$

then the system (48) takes the (nondimensional) form (for scaled prey and adult predators, respectively $x$ and $y$ )

$$
\begin{aligned}
& \frac{d x}{d t}=x(t) b(x(t))-\alpha x(t) y(t)-\gamma x(t) \\
& \frac{d y}{d t}=\alpha x(t-r) y(t-r)-y(t) .
\end{aligned}
$$

Notice that in this scaling the transfer from $x$ to $y$ by the scaled predation is again perfectly efficient, and the mortality factor, $e^{-d_{1} R}$, is scaled out. The properties of $b$ take the same form as in (10). The boundary equilibria are again at $(0,0)$ and $(1,0)$. A somewhat simplified version of the proof in the second paragraph of the proof for Lemma 7 gives us

Lemma 12. For every positive solution $(x(t), y(t)), \limsup _{t \rightarrow \infty} x(t) \leq 1$.

Corollary 13. Suppose $\alpha<1$. Then all positive solutions of (50) converge to $(1,0)$ as $t \rightarrow \infty$.

Proof. Let $(x(t), y(t))$ be a positive solution. First we show that $y(t) \rightarrow 0$. If $\beta$ is chosen such that $\alpha<\beta<1$ then by the above lemma there is some $t^{*}$ such that $\alpha x(t-r) \leq \beta$ for all $t \geq t^{*}$ and so

$$
y^{\prime}(t)=\alpha x(t-r) y(t-r)-y(t) \leq \beta y(t-r)-y(t) .
$$

Since $0<\beta<1$, all solutions of

$$
w^{\prime}(t)=\beta w(t-r)-w(t)
$$

tend to zero as $t \rightarrow \infty$ ([5], section 2). Furthermore all solutions of (52) with positive initial data remain positive for all positive $t$. From these considerations we can conclude ([20]) that if a solution $w(t)$ of (52) shares positive initial data with $y(t)$ then $0<y(t) \leq w(t)$ for all $t \geq 0$. So

$$
y(t) \rightarrow 0
$$

as claimed.

Setting $M=\liminf _{t \rightarrow \infty} x(t)>0$, one shows that $M>0$ in exactly the same way as in Corollary 6 . Now we show $M=1$. (Recall $b(1)=\gamma$.) Suppose not. Then by the previous lemma, $M \leq 1$, so $0<M<1$. Let $M_{1}$ be such that $M<$ $M_{1}<1$. Then $b\left(M_{1}\right)-\gamma>b(1)-\gamma=0$. By (53), for all $t$ larger than some $T_{1}$, 
we have $0<\alpha y(t)<\left(b\left(M_{1}\right)-\gamma\right) / 2$ and $x(t) \geq M / 2$. Hence whenever $t>T_{1}$ and $\frac{1}{2} M \leq x(t) \leq M_{1}$, we have

$$
\begin{aligned}
x^{\prime}(t) & =x(t)(b(x(t))-\alpha y(t)-\gamma) \\
& \geq \frac{M}{2}\left(b\left(M_{1}\right)-\frac{\left(b\left(M_{1}\right)-\gamma\right)}{2}-\gamma\right) \\
& =\frac{M}{2}\left(b\left(M_{1}\right)-\gamma\right) / 2
\end{aligned}
$$

providing a uniform positive lower bound on $x^{\prime}(t)$ whenever $t$ is large and $\frac{1}{2} M \leq$ $x(t) \leq M_{1}$ if $M<1$, another contradiction. Hence $M=1$ and

$$
\liminf _{t \rightarrow \infty} x(t)=\limsup _{t \rightarrow \infty} x(t)=\lim _{t \rightarrow \infty} x(t)=1,
$$

as desired.

Finally we consider the case $\alpha>1$. (For an interpretation of $\alpha$, see section 6.) In this case $b(1 / \alpha)>b(1)=\gamma$ and there is a positive equilibrium $\left(x^{*}, y^{*}\right)$,

$$
\begin{aligned}
& x^{*}=1 / \alpha, \\
& y^{*}=(b(1 / \alpha)-\gamma) / \alpha .
\end{aligned}
$$

To simplify notation somewhat, we let $b_{*}=b\left(x^{*}\right)$ and $b_{*}^{\prime}=b^{\prime}\left(x^{*}\right)$. Note that $b_{*}>\gamma$ and $b_{*}^{\prime}<0$ and that both $b_{*}$ and $b_{*}^{\prime}$ depend on $\alpha$.

THEOREM 14. Consider (50) in the case $\alpha>1$, that is, in the case that there is an interior equilibrium $\left(x^{*}, y^{*}\right)$.

1. If $\alpha\left(b_{*}-\gamma\right)+2 b_{*}^{\prime}<0$ the equilibrium $\left(x^{*}, y^{*}\right)$ is linearly stable for all delays $r \geq 0$

2. If $\alpha\left(b_{*}-\gamma\right)+2 b_{*}^{\prime}>0$ then there is a critical delay $r_{1}>0$ such that the equilibrium $\left(x^{*}, y^{*}\right)$ is linearly stable for all delays $0 \leq r<r_{1}$ and is linearly unstable for all delays $r>r_{1}$. A Hopf bifurcation occurs as $r$ increases through $r_{1}$.

Proof. The linearization of (50) about $\left(x^{*}, y^{*}\right)$ is

$$
\begin{aligned}
u^{\prime}(t) & =x^{*} b_{*}^{\prime} u(t)-\alpha x^{*} v(t) \\
& =b_{*}^{\prime} u(t) / \alpha-v(t) \\
v^{\prime}(t) & =\alpha y^{*} u(t-r)+\alpha x^{*} v(t-r)-v(t) \\
& =\left(b_{*}-\gamma\right) u(t-r)+v(t-r)-v(t),
\end{aligned}
$$

yielding the linear equation for solutions of the form $(u(t), v(t))=e^{z t}\left(u_{0}, v_{0}\right)$,

$$
z e^{z t}\left[\begin{array}{l}
u_{0} \\
v_{0}
\end{array}\right]=e^{z t}\left[\begin{array}{cc}
b_{*}^{\prime} / \alpha & -1 \\
\left(b_{*}-\gamma\right) e^{-z r} & \left(e^{-z r}-1\right)
\end{array}\right]\left[\begin{array}{l}
u_{0} \\
v_{0}
\end{array}\right]
$$

and the characteristic equation

$$
\begin{aligned}
0 & =\operatorname{det}\left[\begin{array}{cc}
b_{*}^{\prime} / \alpha-z & -1 \\
\left(b_{*}-\gamma\right) e^{-z r} & e^{-z r}-1-z
\end{array}\right] \\
& =\frac{1}{\alpha}\left(-b_{*}^{\prime}-\left(b_{*}^{\prime}-\alpha\right) z+\alpha z^{2}+e^{-z r}\left(b_{*}^{\prime}+\alpha b_{*}-\alpha \gamma-\alpha z\right)\right)
\end{aligned}
$$

or

$$
0=\alpha z^{2}-\left(b_{*}^{\prime}-\alpha\right) z-b_{*}^{\prime}+e^{-z r}\left(-\alpha z+\alpha b_{*}+b_{*}^{\prime}-\alpha \gamma\right) .
$$


We apply the results of Cooke and van den Driessche [7] to analyze the roots of the characteristic equation. In their notation, we set

$$
\begin{aligned}
& P(z)=\alpha z^{2}-\left(b_{*}^{\prime}-\alpha\right) z-b_{*}^{\prime}, \\
& Q(z)=-\alpha z+\alpha\left(b_{*}-\gamma\right)+b_{*}^{\prime} .
\end{aligned}
$$

According to [7], $z=i y$ is a purely imaginary characteristic root only if $F(y)=0$ where $F(y)=|P(i y)|^{2}-|Q(i y)|^{2}$, and furthermore:

1. If $F(y)=0$ has no positive roots and the equilibrium is stable when $r=0$, then it is stable for all $r>0$.

2. If $F(y)=0$ has a positive root and each positive root is simple, then there is an $r_{1}$ such that the equilibrium is unstable for $r>r_{1}$ and the direction of a characteristic root crossing the imaginary axis is given by the sign of $F^{\prime}(y)$.

To apply these results, we find

$$
\begin{aligned}
F(y) & =\left|-\alpha y^{2}-\left(b_{*}^{\prime}-\alpha\right) i y-b_{*}^{\prime}\right|^{2}-\left|-\alpha i y+\alpha\left(b_{*}-\gamma\right)+b_{*}^{\prime}\right|^{2} \\
& =\alpha^{2} y^{4}+b_{*}^{\prime 2} y^{2}-\alpha^{2} b_{*}^{2}+2 \alpha^{2} b_{*} \gamma-2 \alpha b_{*} b_{*}^{\prime}-\alpha^{2} \gamma^{2}+2 \alpha b_{*}^{\prime} \gamma .
\end{aligned}
$$

Let's examine the constant term of $F(y)$,

$$
\alpha\left(-\alpha b_{*}^{2}+2 \alpha b_{*} \gamma-2 b_{*} b_{*}^{\prime}+2 b_{*}^{\prime} \gamma-\alpha \gamma^{2}\right)=-\alpha\left(b_{*}-\gamma\right)\left(2 b_{*}^{\prime}+\alpha\left(b_{*}-\gamma\right)\right) .
$$

As mentioned just before this theorem, the assumption of $\alpha>1$ gives us $b_{*}>\gamma$. Since $b_{*}^{\prime}<0$ the sign of the factor $2 b_{*}^{\prime}+\alpha\left(b_{*}-\gamma\right)$ can vary depending on the implementation of $b(x)$.

Clearly if $2 b_{*}^{\prime}+\alpha\left(b_{*}-\gamma\right)<0$, then $F$ has no real zeros and so the characteristic equation has no purely imaginary roots. According to [7], since the equilibrium is stable under zero delay (Theorem 4 ), it is stable under all nonnegative delays.

However, if $2 b_{*}^{\prime}+\alpha\left(b_{*}-\gamma\right)>0$, then $F$ will have exactly one positive zero, corresponding to a characteristic root with positive imaginary part. In this case the direction of a characteristic root crossing the imaginary axis is given by the sign of $F^{\prime}(y)[7]$ which is positive when $y$ is positive. So any crossing is transverse, from left to right. The second conclusion of the theorem now follows exactly from [7] and standard bifurcation theory (e.g. [14], p. 332).

Since Theorem 14 concerns the dimensionless system (50) obtained by scaling out the physical presence of the delay from (48), we should explain how this theorem can be applied to the dimensional system (48). First note that $\alpha>1$ is equivalent to $d_{P} e^{d_{1} R} / c<N_{0}$, so that the latter is equivalent to the existence of a positive equilibrium. Upon a substitution of the original parameters into the inequality of part 1 in Theorem 14, we conclude that the positive equilibrium of system (48) exists and is linearly stable if $d_{P} e^{d_{1} R} / c<N_{0}$ and $c e^{-d_{1} R} N_{0}\left(B\left(d_{P} e^{d_{1} R} / c\right)-d\right)+$ $2 B^{\prime}\left(d_{P} e^{d_{1} R} / c\right) N_{0} d_{P}<0$.

However, for the bifurcation at $F=0$ one must proceed cautiously when translating the results from the nondimensional case of Theorem 14 to the dimensional case which motivates it. We naturally inquire if a bifurcation, such as given by Theorem 14 in the nondimensional case with $r$ increasing, is mirrored by one in the corresponding original system (48) with $R$ as the bifurcation parameter and all other parameters (except $r$ ) held fixed. However, such variation of parameters is inherently contradictory! Since

$$
\alpha=c e^{-d_{1} R} N_{0} / d_{P}
$$


we see that if we seek bifurcation in the original system (48) with only $R$ varying, then we must examine Theorem 14 as $\alpha$ varies, instead of relying on it to be constant, with $\alpha>1$, as assumed in the hypotheses of the theorem. In particular, if we increase $R$ while holding fixed all the other parameters in (48), then $\alpha=c e^{-d_{1} R} N_{0} / d_{P}$ must decrease, eventually violating the theorem's hypothesis of $\alpha>1$.

Fortunately, a bifurcation diagram for system (48) can be calculated in the $(R, c)$ parameter plane (while all parameters other than $R, c$ are fixed). To proceed with this for each given $\alpha>0$, let the curve $\Gamma_{\alpha}$ be the level curve of $(56)$ in the $(R, c)$ plane, i.e., the graph of the function

$$
c=\alpha d_{P} e^{d_{1} R} / N_{0}, \quad(R \geq 0)
$$

with $\left(d_{1}, d_{P}, N_{0}\right)$ fixed. There is a positive equilibrium corresponding to some $(R, c)$ on $\Gamma_{\alpha}$ if and only if $\alpha>1$. For such $\alpha$, let $r_{1}=r_{1}(\alpha)$ be the unique positive value defined in part 2 of Theorem 14 and let $R_{1}=R_{1}(\alpha)=r_{1}(\alpha) / d_{p}$. Then from Theorem 14 it immediately follows that such an equilibrium is linearly stable (respectively, unstable) if $R<R_{1}$ (respectively, $R>R_{1}$ ). Moreover, a Hopf bifurcation occurs as the point $(R, c)$ passes through $\left(R_{1}, c_{1}\right)$ along $\Gamma_{\alpha}$, where $c_{1}=c_{1}(\alpha)=\alpha d_{P} e^{d_{1} R_{1}(\alpha)} / N_{0}$.

Let $K(\alpha)$ denote the critical quantity distinguishing the cases of Theorem 14:

$$
K(\alpha)=\alpha(b(1 / \alpha)-\gamma)+2 b r(1 / \alpha) \quad(\alpha>1) .
$$

It is clear that

$$
K(\alpha) \rightarrow+\infty \quad \text { as } \quad \alpha \rightarrow \infty .
$$

Let us first consider the simple case in which there is a unique $\alpha^{*}>1$ such that $K\left(\alpha^{*}\right)=0$ and $K(\alpha)>0$ for $\alpha>\alpha^{*}$. (In section $6 \alpha^{*}$ exists and is given for birth function $b_{i}$ by $\alpha^{*}=\alpha_{i}, i=1,2,3$.) Then for each $\alpha>\alpha^{*}$, there is a unique bifurcation point $\left(R_{1}(\alpha), c_{1}(\alpha)\right)$ in the curve $\Gamma_{\alpha}$. It is obvious that the bifurcation point $\left(R_{1}(\alpha), c_{1}(\alpha)\right)$ is continous with respect to $\alpha$ (but formulas are supplied below). Thus by varying $\alpha$ from $\alpha^{*}$ to $\infty$ we obtain a simple bifurcation curve in the $(R, c)$ parameter plane that does not intersect itself. In what follows we shall show that the function $R_{1}(\alpha)$ has a nice property that

$$
\lim _{\alpha \rightarrow \alpha^{*}} R_{1}(\alpha)=+\infty, \quad \lim _{\alpha \rightarrow \infty} R_{1}(\alpha)=0 .
$$

One readily finds closed form expression for the bifurcation delay $r_{1}$ in the nondimensional equations (50) as it depends on $\alpha$. When $K(\alpha)>0$ the function $F$ in the proof of Theorem 14 has a unique positive zero $y_{0}$ and $r_{1}$ is the least positive solution of $P+e^{-i y_{0} r} Q=0$. Thus, remembering that $P, Q, F, y_{0}$ depend on $\alpha, r_{1}$ is a composition of two functions:

$$
y_{0}(\alpha)=\sqrt{\frac{1}{2 A}\left(-B+\sqrt{B^{2}-4 A C}\right)},
$$

where

$$
A=\alpha^{2}, \quad B=b^{\prime}(1 / \alpha)^{2}, \quad C=-\alpha(b(1 / \alpha)-\gamma)\left(2 b^{\prime}(1 / \alpha)+\alpha(b(1 / \alpha)-\gamma)\right),
$$

followed by

$$
r_{1}(\alpha)=\rho(\alpha)=\frac{1}{y_{0}(\alpha)} \arg \left(\frac{-Q\left(i y_{0}(\alpha), \alpha\right)}{P\left(i y_{0}(\alpha), \alpha\right)}\right)
$$


where arg denotes the argument of a complex number. Using the expressions of $P, Q$ one is able to verify that for all sufficiently large $\alpha$,

$$
0<\arg \left(\frac{-Q\left(i y_{0}(\alpha), \alpha\right)}{P\left(i y_{0}(\alpha), \alpha\right)}\right)<\pi .
$$

Furthermore, (57) and (58) yield that

$$
\lim _{\alpha \rightarrow \infty} y_{0}(\alpha)=\sqrt{b(0)-\gamma}, \quad \lim _{\alpha \rightarrow \infty} \frac{-Q\left(i y_{0}(\alpha), \alpha\right)}{P\left(i y_{0}(\alpha), \alpha\right)}=1
$$

and it follows from (59) and (61) that

$$
\lim _{\alpha \rightarrow \infty} R_{1}(\alpha)=\frac{1}{d_{P}} \lim r_{1}(\alpha)=\frac{1}{d_{P} \sqrt{b(0)-\gamma}} \arg (1)=0 .
$$

Finally we easily see that

$$
\lim _{\alpha \rightarrow \alpha^{*}} y_{0}(\alpha)=0, \quad \lim _{\alpha \rightarrow \alpha^{*}} \frac{-Q\left(i y_{0}(\alpha), \alpha\right)}{P\left(i y_{0}(\alpha) \cdot \alpha\right)}=-1
$$

which implies that

$$
R_{1}(\alpha) \rightarrow+\infty \quad \text { as } \quad \alpha \searrow \alpha^{*} .
$$

With the use of (62) and (64) we can describe the bifurcation diagram of the dimensional system $(48)$ in the $(R, c)$ plane (see Figure 1$)$. The positive quadrant of the $(R, c)$ plane is divided into three parts by the curves $\Gamma_{1}: c=d_{P} e^{d_{1} R} / N_{0}$ and $\Gamma=\left\{\left(R_{1}(\alpha), c_{1}(\alpha)\right): \alpha \in\left(\alpha^{*}, \infty\right)\right\}$. The curve $\Gamma$ is asymptotic to the graph $\Gamma_{\alpha^{*}}: c=\alpha^{*} d_{P} e^{d_{1} R} / N_{0}$ as $\alpha \searrow \alpha^{*}$ and is asymptotic to the vertical line $R=0$ as $\alpha \rightarrow \infty$. Region I corresponds to system (48) having no positive equilibrium; region II corresponds to system (48) having a stable positive equilibrium; and region III corresponds to system (48) with an unstable positive equilibrium. $\Gamma$ is a hopf bifurcation curve.

We finally remark that if there is any pair of two numbers $1<\alpha_{1}<\alpha_{2}$ such that $K\left(\alpha_{1}\right)=K\left(\alpha_{2}\right)=0$, with $K(\alpha)>0, \alpha \in\left(\alpha_{1}, \alpha_{2}\right)$ then the curve $\left\{\left(R_{1}(\alpha), c_{1}(\alpha)\right)\right.$ : $\left.\alpha \in\left(\alpha_{1}, \alpha_{2}\right)\right\}$ gives rise to a Hopf bifurcation curve that is asymptotic to the graph $\Gamma_{\alpha_{i}}$ as $\alpha \rightarrow \alpha_{i}$ for $i=1,2$.

The parameterization of $\Gamma,\left(R_{1}(\alpha), c_{1}(\alpha)\right)$ can be found with $R_{1}(\alpha)$ explicitly and $c_{1}(\alpha)$ explicitly, using (59) and $c=\alpha d_{P} e^{d_{1} R} / N_{0}$. Since

$$
R_{1}=r_{1} / d_{P}=\rho(\alpha) / d_{P}=\rho\left(c e^{-d_{1} R_{1}} N_{0} / d_{P}\right) / d_{P},
$$

we have

$$
\begin{aligned}
R_{1}(\alpha) & =r_{1}(\alpha) / d_{P}=\rho(\alpha) / d_{P}, \\
c_{1}(\alpha) & =d_{p} e^{d_{1} R_{1}(\alpha)} \sigma\left(d_{P} R_{1}(\alpha)\right) / N_{0}
\end{aligned}
$$

if $\sigma$ inverts $\rho$. When one has $\rho$ numerically, $\sigma$ just reverses the coordinates, so this is easy to plot.

We consider two examples. Since the physical presence of the delay is a key feature in (48), we simplify everything else as much as possible (but with some minimal care to respect biological interpretation of the parameters: see below), taking

$$
a=d_{1}=d_{P}=N_{0}=1,
$$




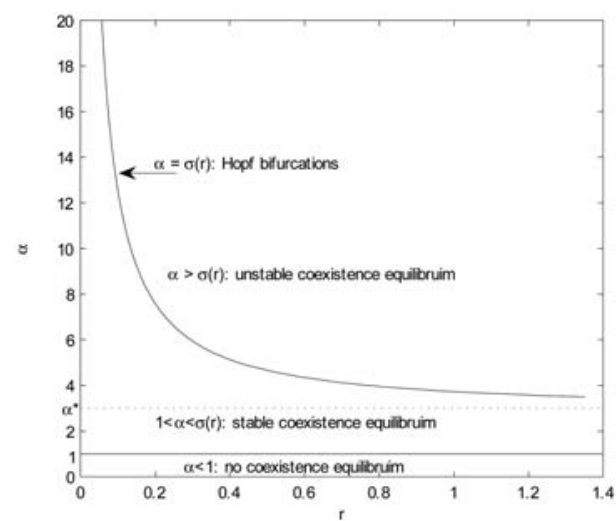

(a)

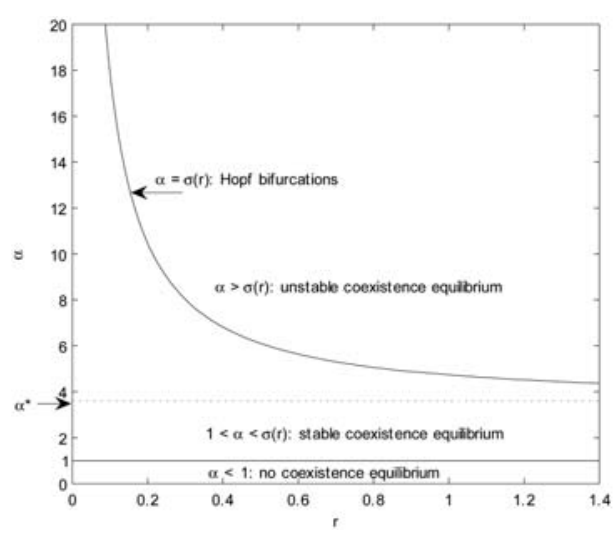

(c)

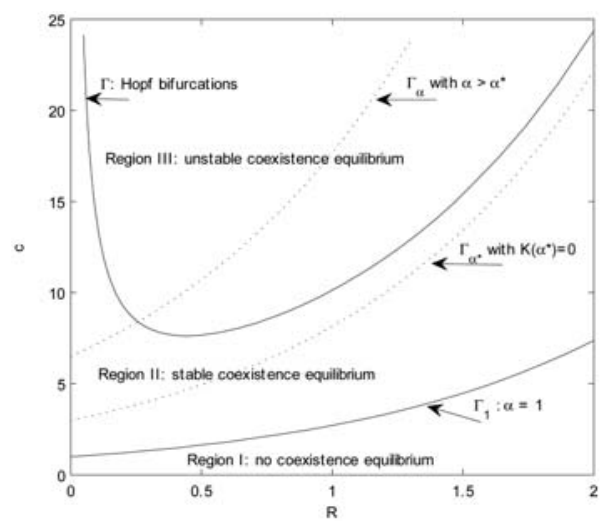

(b)

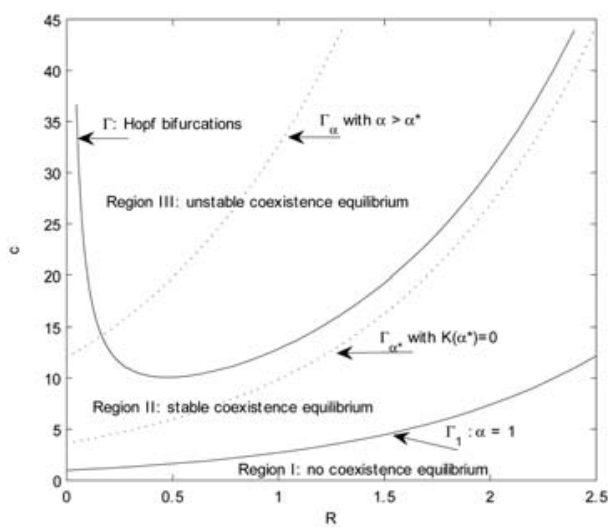

(d)

FIG. 1. (a) Scaled system, $b(x)=8(1-x / 4)$; (b) Dimensional system, $B(X)=8(1-X / 4)$; (c) Scaled system, $b(x)=9 /(1+0.5 x)$; (d) Dimensional system, $B(X)=9 /(1+0.5 X)$.

so that in the corresponding dimensionless system

$$
\alpha=c e^{-R}, \quad t=T, \quad x(t)=N(t), \quad y(t)=e^{R} P(t) / c, \quad b(x)=B(x) .
$$

In the case of $B(X)=8(1-X / 4)$ giving $\gamma=b(1)=6$, we find bifurcation diagrams as in (a) and (b) of Figure 1 , and then, when $B(X)=9 /(1+0.5 X)$, again with $\gamma=b(1)=6$, we find bifurcation diagrams as in (c) and (d) of Figure 1.

Notice in both examples that for appropriately large, fixed $c$ with $R$ increasing from 0 the dimensional system (48) goes first through a supercritical Hopf bifurcation, and then back through a subcritical one.

\section{Numerical examples.}

6.1. Interpretation of parameters. In choosing parameter values for numerical examples, it is helpful to think about the ideas they represent. Thus we consider:

1. $t$ which is time scaled by $1 / d_{P}$, which we may take as a measure of the expected lifespan for predators.

2. $\alpha$, which has been a focus of attention, beginning with the condition $\alpha>1$ as our condition for the existence of an interior equilibrium at $\left(x^{*}, y^{*}\right)=$ 
$(1 / \alpha,(b(1 / \alpha)-\gamma) / \alpha)$, and the key to an additional condition for a Hopf bifurcation at this equilibrium. We find that $\alpha$ is similar to $R_{0}$ in some ODE models, by first observing that in a complete absence of predators, $N$ is steady at $N_{0}$ which functions as its carrying capacity. Then, if a very small number of predators is introduced and holds roughly constant (near the equilibrium), we find $c e^{-d_{1} R}-d_{P}$ to be the net per unit rate of change of $P$ and hence $\alpha=c e^{-d_{1} R} N_{0} / d_{P}$ is the number of newly matured predators per adult predator, produced during a predator lifetime at prey carrying capacity. Thus we may interpret it as a replacement ratio and indeed similar to $R_{0}$. Likewise, $1 / \alpha$ can be thought of as the fraction of an average adult predator's lifetime needed for self-replacement, at prey carrying capacity. All such considerations are with respect to a common unit of measure for predator and prey, for example, biomass.

3. $\gamma=d / d_{P}$, which is $b(1)$ in consequence of our scaling of $x$. Similarly to interpreting $1 / d_{P}$ above, we find that $\gamma$ is the ratio of the lifetime of a predator to that of a prey. In scenarios such as the present in which maturation time of predators is deemed important to include in the model, while that of prey is not, we expect $\gamma>1$.

4. $b\left(x^{*}\right) / b(1)=B\left(x^{*} N_{0}\right) / d$ is the prey lifetime recruitment (per unit) at equilibrium.

6.2. Comparison of birth functions: Affine, concave up, and concave down. In this section we numerically investigate three implementations of the birth function,

$$
\begin{aligned}
& b_{1}(x)=a\left(1-\frac{x}{b}\right), \\
& b_{2}(x)=\frac{c}{1+d x} \\
& b_{3}(x)=p\left(1+\frac{1}{x-q}\right),
\end{aligned}
$$

in order to better understand the behavior generally of the nondimensional models from section 5, especially with regard to the bifurcations guaranteed by Theorem 14 . We determine the pairs of coefficients $((a, b),(c, d),(p, q))$ so that the implementations of the $b_{i}$ have the same interior equilibrium $\left(x^{*}, y^{*}\right)$, the same value of $b_{i}\left(x^{*}\right)$ and the same model coefficients $\alpha, \gamma$, and with values so that the conditions for Hopf bifurcation are satisfied. Notice that over the domain of interest, $b_{1}$ is affine; $b_{2}$ is concave up; and $b_{3}$ is concave down. Although $b_{1}$ does not satisfy the condition $(x b(x))^{\prime} \geq 0$ if $x>b / 2$, we use it only on $0<x<b / 2$ where that condition is satisfied. The function can be redefined elsewhere to satisfy the condition if desired, with no effect on our computations. All the numerical computations use the Matlab-based package DDE-BIFTOOL [18].

Implementation. The interpretations above guide us in choosing values for $\beta=$ $b\left(x^{*}\right)$ and $\gamma=b(1)$. The parameter $\alpha$ determines the equilibrium $\left(x^{*}, y^{*}\right)$ through

$$
\begin{aligned}
& x^{*}=1 / \alpha \\
& y^{*}=\left(b\left(x^{*}\right)-b(1)\right) x^{*}=(b(1 / \alpha)-\gamma) / \alpha .
\end{aligned}
$$

We then solve for the two parameters in each of the birth functions $b_{i}$. There are elementary but tedious details to be checked that all the requirements on the birth function and $\alpha$ are satisfied, which we omit. The resulting restrictions are listed here. 
Proposition 15. For $i=1,2,3$, there is an $\alpha_{i}>1$ such that the condition (2) of Theorem 14 for bifurcation in the model based on $b_{i}$ is satisfied if and only if $\alpha>\alpha_{i}$. (Thus the $\alpha_{i}$ satisfy the criteria of $\alpha^{*}$ above.) Furthermore, whenever $\beta>\gamma>0$ and the restrictions immediately below hold, positive parameters for the birth function $b_{i}$ are uniquely determined such that with $x^{*}=1 / \alpha<1 / \alpha_{i}$ we have $b_{i}\left(x^{*}\right)=\beta$ and $b_{i}(1)=\gamma$.

Table 1 summarizes the relevant relations and conditions.

TABLE 1

\begin{tabular}{|c|c|}
\hline \multirow{3}{*}{$b_{1}(x)=a\left(1-\frac{x}{b}\right)$} & $\begin{array}{c}a=\frac{\alpha \beta-\gamma}{\alpha-1}, b=\frac{\alpha \beta-\gamma}{\alpha(\beta-\gamma)} \\
(x b(x))^{\prime}>0 \text { if } \gamma<\beta<2 \gamma \\
\alpha_{1}=3\end{array}$ \\
\hline \multirow{2}{*}{$b_{2}(x)=\frac{c}{1+d x}$} & $c=\beta \frac{\alpha-1}{\alpha-\beta / \gamma}, d=\alpha \frac{\beta / \gamma-1}{\alpha-\beta / \gamma}$ \\
& Both $c, d$ are positive iff $\alpha>\beta / \gamma>1$. \\
& $\alpha_{2}=\left(3+d+\sqrt{9+10 d+d^{2}}\right) / 2$ \\
\hline$b_{3}(x)=p\left(1+\frac{1}{x-q}\right)$ & $p=$ larger zero of $(\alpha-1) Z^{2}-(\beta(2 \alpha-1)-\gamma) Z+(\alpha-1) \beta \gamma$ \\
& $(x=(\beta-p-p \alpha) /(\alpha(\beta-p))$ \\
& $(x b)^{\prime}>0$ if $q>\frac{3}{2}+\frac{1}{2} \sqrt{5} \approx 2.62$ \\
& $\alpha=\frac{1}{2 q}\left(3 q-1+\sqrt{9 q^{2}-10 q+1}\right)$ \\
\hline
\end{tabular}

Discussion. We saw above that we must respect $0<\gamma<\beta$, while numerically we observed that this together with $\beta \leq 3 \gamma / 2$ was necessary and sufficient for each $b_{i}$ to be implemented over an interval for $\alpha$ including $\left[\alpha_{i}, 7\right]$ in its interior. For each $b_{i}$ in combination with each of the pairs $(\beta, \gamma)=(4.5,3),(8,6)$ we computed a locus of Hopf bifurcation points in the $(\alpha, r)$ plane which included $5 \leq \alpha \leq 7$ in all cases. Then in each of the cases $\alpha=5,7$, we computed bifurcation diagrams with the delay $r$ as bifurcation parameter, and plotted: (1) bifurcation diagrams, (2) profiles of the bifurcating periodic solutions (traces of the solutions $(x(t), y(t))$ ), and (3) the largest size of a nontrivial Floquet multiplier. (See [6] for more extensive graphics.) These computations were done for values of the delay $r$ beginning at the bifurcation value and ending at about $r=2$. Considering that we scaled time by the measure of a predator lifetime, $1 / d_{P}$, a delay of $r=2$ corresponds to a juvenile predator maturation that is twice this magnitude, and hence is more than adequate for biological considerations.

There were remarkable similarities across these computations.

1. All the bifurcation loci in the $(\alpha, r)$ plane were decreasing, concave up.

2. All the bifurcation diagrams of the $x$-amplitude of bifurcating period solutions vs. $r$ were increasing, concave down, with the amplitude approaching 1 as $r$ increased. (Recall that the "carrying capacity" for $x$ is normalized to 1.)

3. All the bifurcation diagrams of the period of bifurcation periodic solutions vs. $r$ were increasing and almost linear for a long range of $r$. However, this broke down as discussed below.

4. All the profile plots of the periodic solutions (that is, projections of periodic solutions $(x(t), y(t)$ into an $(x, y)$ plane) showed initial nesting of each surrounding those of lesser $r$, expanding with increasing $r$ but contained within a triangle adjacent to the origin and coordinate axes in the $(x, y)$ plane, and then developing an "overhang." See Figure 2 for a typical scenario.

5. Floquet multipliers (These are eigenvalues of the linearized Poincaré map associated with each bifurcating periodic solution and always "trivially" in- 


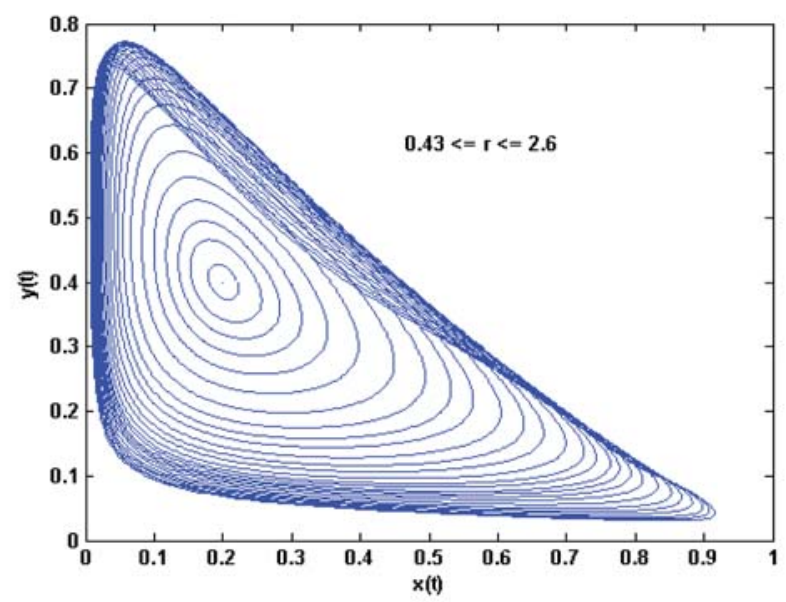

FIG. 2. Overhang in bifurcating family of periodic solution profiles.

clude 1 corresponding to the initial condition of the periodic solution. Only a finite number can lie outside any disk centered at the origin in the complex plane.): In plotting the largest magnitude of these (excepting the trivial 1) vs. $r$, we found rapid decrease to small values across all our examples, followed in some circumstances by eventual increase (but not in ranges of $r$ that are biologically compelling).

Prompted by the eventual increase of Floquet multipliers for $r>2$ we singled out the example case of birth function $b_{1}$ with $\alpha=5, \beta=8, \gamma=6$ for further investigation and extended the delay $r$ from the bifurcation value of about 0.43 to over 8 . Some interesting aspects emerged, which although not of direct biological interest in the current context, might easily emerge in others. They include:

1. Self-crossover of periodic orbit profiles: At $r \approx 3.8$ we observe the beginnings of self-intersection within profiles of the bifurcating periodic orbits. See the sequence in Figure 3. Since the periods of these solutions increased approximately affinely with the delay over this regime, perhaps this extra looping of the profile can be understood as a mechanism by which the longer period is accommodated in a limited spatial region. There were no remarkable aspects of the Floquet multipliers (measures of stability) apparently associated with these behaviors.

2. The profiles of the self-intersecting orbits also approach the equilibrium at $(1,0)$ (thereby slowing down) and develop folds and spikes in their upper left corner, again with the effect of enabling longer periods. See the sequence in Figure 3.

3. It is quite remarkable that over $0.43<r<8$ the bifurcation diagram of period vs. $r$ is approximately affine with the notable exception of $7.12<r<7.29$ where it doubles over in a backwards S. Moreover, the vertical tangencies (in the period vs. delay bifurcation diagrams) at $r=7.12,7.29$ are accompanied by a Floquet multiplier leaving the unit disk in the complex plane. Notice that the self-crossovers remarked on above appear to be completely independent of the branch of multipliers that leaves the unit disk over $4.8<r<6.3$. See Figure 4. 


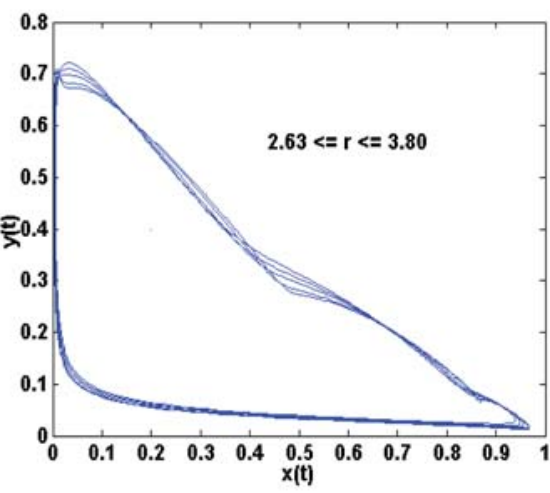

(a)

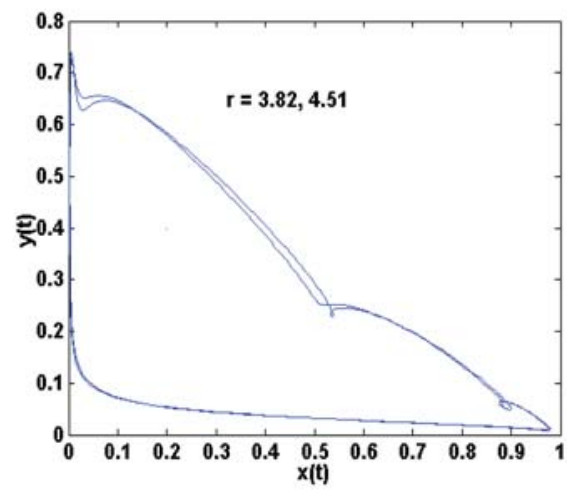

(b)

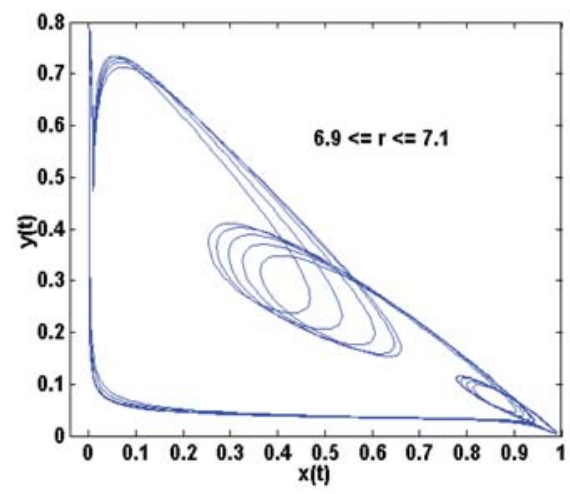

(c)

FIG. 3. Progression of self-intersecting profiles of bifurcating period orbits. (a) As $r$ increases to 3.8, a cusp develops (lower right) and early stages of a fold are seen (upper left). (b) Intermediate stages of both self-intersection and fold. (c) Late stages at large $r$.

7. Equivalence of dimensional and nondimensional results. There are some concerns that might arise concerning the scaling of the original models (5) in the host-parasite case (and (48) in the predator-prey case) and how analysis of the resulting dimension-free resulting models (respectively, (9) and (50)) applies back to those original models. In particular, there might be some concern about the use of the scaled delay, $r$, as a bifurcation parameter in (50) after a coefficient in (48) which "physically" contained the original delay, $R$, has been scaled out. Furthermore, there might arise concern about the validity of the bifurcation analysis since the dimension of the parameter space is reduced from eight (including the delay and $N_{0}$ ) in the original models to three (including the scaled delay) in the corresponding dimensionfree ones. Since the relation between (48) and (50) scales out the "physical" presence of the delay and is therefore more complicated, we will focus on that situation, leaving the other host-parasite situation of section 3 as an easy corollary. We should note that Beretta and Kuang [1] address these issues without passing to a scaled version of the model. Our situation, however, can be addressed directly.

Let us refer to $(48 \mathrm{a}-48 \mathrm{c})$, together with the assumptions $(2 \mathrm{a}),(2 \mathrm{~b})$, and $(7)$, as the dimensional model. Recall that we defined $N_{0}$ by the condition $B\left(N_{0}\right)=d$. Then 

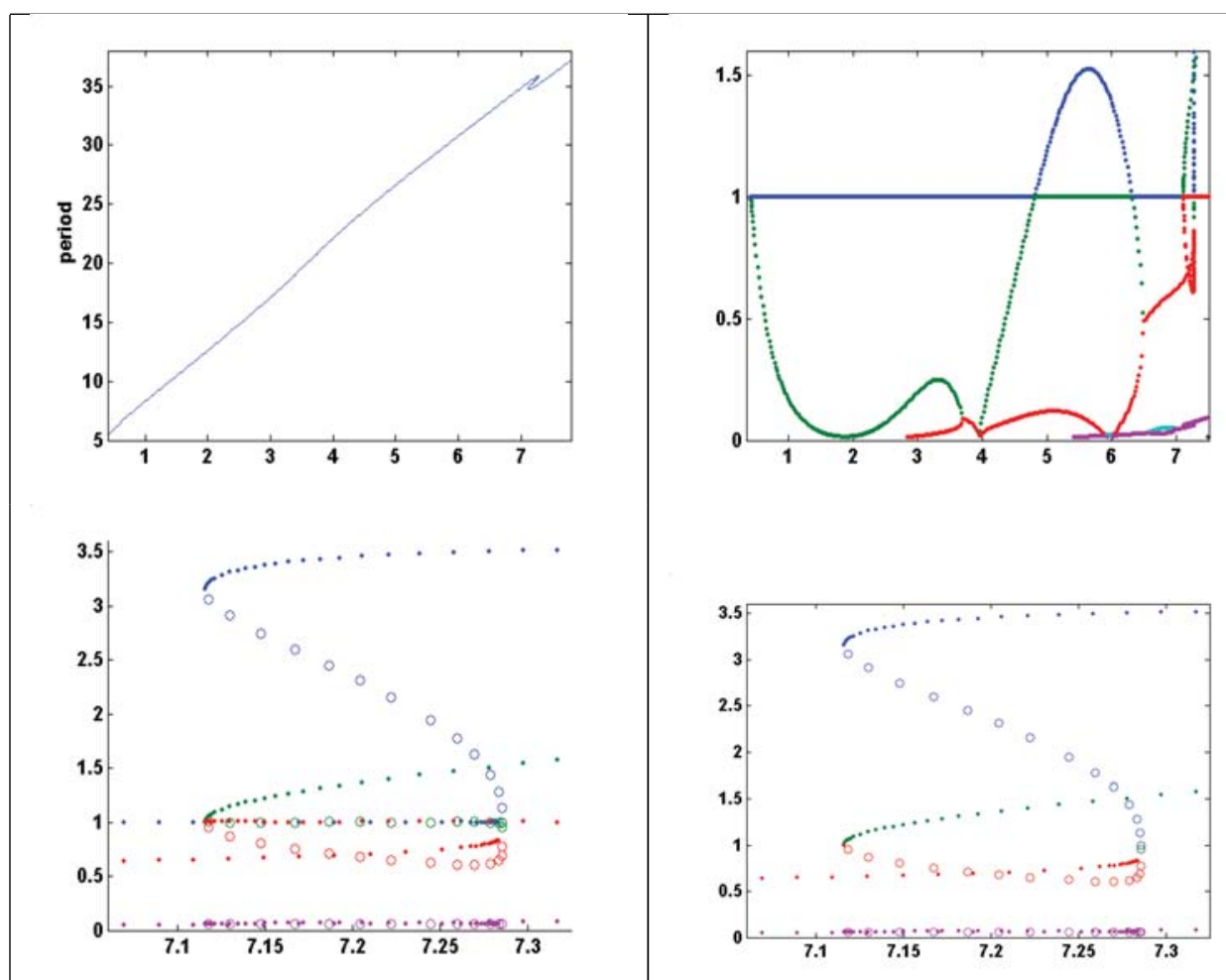

FIG. 4. With the maturation delay, $r$, on the horizontal axis: Upper left shows periods, including the backwards S. All other frames show absolute values of Floquet multipliers larger than 0.01: Upper right shows values $<7.5$. Lower frames have dots plotted as both $r$ and the period increase, first over $7.05<r<7.29$ (corresponding to the top of the backwards $S$ in the bifurcation diagram), and later over $7.12<r<7.35$ (corresponding to the bottom of the backwards $S$ ). To contrast circles are plotted as $r$ decreases from 7.29 to 7.12 through the middle of the backwards $S$. The lower right is the same as lower left with values of 1 removed to fascilitate observation of a branch of complex conjugate multipliers (shown only in absolute value) becoming real at $r=7.29$, with one leaving the unit disk there, and the other leaving after $r$ decreases to 7.12 .

we scaled variables and parameters according to (49), resulting in the nondimensional model

$$
\begin{aligned}
& \frac{d x}{d t}=x(t) b(x(t))-\alpha x(t) y(t)-\gamma x(t), \\
& \frac{d y}{d t}=\alpha x(t-r) y(t-r)-y(t),
\end{aligned}
$$

satisfying

$$
b(1)=\gamma
$$

The purpose of this section is to show that bifurcation in the latter system (68) with respect to $r$ implies bifurcation in the former, (48), with respect to $R$, and conversely.

Suppose now that $(\alpha, \gamma, r, b(\cdot))$ are given and consider the corresponding system (68). Also consider a system (48) in which $\left(a, c, d, d_{1}, d_{P}, N_{0}, R, B(\cdot)\right)$ are given such 
that the parametric relations of (49) hold, namely,

$$
\begin{aligned}
c e^{-d_{1} R} N_{0} / d_{P} & =\alpha, \\
d / d_{P} & =\gamma, \\
d_{P} R & =r, \\
B\left(x N_{0}\right) / d_{P} & =b(x) .
\end{aligned}
$$

Then, given a solution $(x(t), y(t))$ of $(68)$, if

$$
\begin{aligned}
T & =t / d_{P}, \\
N(T) & =N_{0} x\left(d_{P} T\right), \\
P(T) & =d_{P} \alpha y\left(d_{P} T\right) / a,
\end{aligned}
$$

it is easy to see that $(N(T), P(T))$ solves (48). In this way (while parameters are fixed and appropriately related), solutions of the two systems are related by a bicontinuous linear isomorphism. In this context, stability of equilibria and periodic solutions carries over from (68) to (48), and conversely.

In examining implications for bifurcation, we have already seen that it is not possible with our choice of scaling to vary only the delays, $r$ and $R$. However, suppose again that $(\alpha, \gamma, r, b(\cdot))$ are given with $(\alpha, \gamma, b(\cdot))$ fixed, but with $r$ varying, and let $\left(c, d, d_{1}, d_{P}, N_{0}, B(\cdot)\right)$ be smooth functions of $R=r / d_{P}$ such that $(69)$ and $B\left(N_{0}\right)=d$ continue to hold. Again, given a solution $(x(t), y(t))$ of (68), if (69) holds it is easy to see that $(N(T), P(T))$ solves (48) for each value of $r$ with $R=r / d_{P}$. Moreover the triples $(x(\cdot), y(\cdot), r)$ and $(N(\cdot), P(\cdot), R)$ are related by a bicontinuous bijection, based on the bicontinuous linear isomorphism between $(x(\cdot), y(\cdot))$ and $(N(T), P(T))$ above, but with nonlinear inclusion of $r$. This correspondence maintains equilibrium, periodicity, and stability properties of solutions, so that if a bifurcation occurs in (68) with respect to $r$, then a corresponding one in (48) must also occur with respect to $R$.

To address the converse question regarding implications of bifurcations in (48) for bifurcations in (68), let us consider the case of $R$ as bifurcation parameter in (48) with $\left(a, c, d, d_{1}, d_{P}, N_{0}\right)$ held fixed. Then assuming our usual relations between that system and $(68)$, we again have the triples $(N(\cdot), P(\cdot), R)$ and $(x(\cdot), y(\cdot), r)$ related by a bicontinuous bijection that preserves equilibrium, periodicity, and stability properties. Thus any bifurcation in (48) with respect to $R$ is mirrored by one in (68) with respect to $r$, but with $\alpha$ also varying (as a function of $r$, through (56)), perhaps with consequences, e.g., for the existence of a coexistence equilibrium as $\alpha$ decreases through unity.

\section{REFERENCES}

[1] Beretta, E. And Y. Kuang, Geometric stability switch criteria in delay differential systems with delay dependent parameters, SIAM J. Math. Anal., 33 (2002), pp. 1144-1165.

[2] C. J. Briggs, R. M. Nisbet, And W. W. Murdoch, Delayed feedback and multiple attractors in a host-parasitoid system, J. Math. Biol., 38 (1999), pp. 317-345.

[3] C. J. Briggs and H. C. J. Godfray, The dynamics of insect-pathogen interactions in stagestructured populations, Amer. Naturalist, 145 (1995), pp. 855-887.

[4] M. Cavani, M. Lizana, And H. Smith, Stable periodic orbits for a predator-prey model with delay, J. Math. Anal. Appl., 249 (2000), pp. 324-339.

[5] K. L. Cooke and Z. Grossman, Discrete delay, distributed delay and stability switches, J. Math. Anal. Appl., 86 (1982), pp. 592-627. 
[6] K. L. Cooke, R. H. Elderkin, And W. Huang, Appendix of graphics, http://www.pages. pomona.edu/ relderkin/pub/GraphAppend.pdf (2004).

[7] K. L. Cooke And P. van Den Driessche, On zeroes of some transcendental equations, Funkcial. Ekvac., 29 (1986), pp. 77-90.

[8] K. L. Cooke, P. van den Driessche, and X. Zou, Interaction of maturation delay and nonlinear birth in population and epidemic models, J. Math. Biol., 39 (1999), pp. 332-352.

[9] J. M. Cushing, Integrodifferential Equations and Delay Models in Population Dynamics, Lecture Notes in Biomath. 20, Springer-Verlag, Berlin/Heidelberg/New York, 1977.

[10] O. Diekmann, S. A. van Gils, S. M. V. Lunel, and H.-O. Walther, Delay Equations: Functional-, Complex-, and Nonlinear Analysis, Springer-Verlag, New York, 1995.

[11] H. Freedman, I. Deterministic Mathematical Models in Population Ecology, 2nd ed., HIFR Consulting, Edmonton, 1987.

[12] K. Gopalsamy, Stability and Oscillations in Delay Differential Equations of Population Dynamics, Kluwer Academic Publishers, Dordrecht/Boston/London, 1992.

[13] S. A. Gourley and Y. Kuang, A stage structured predator-prey model and its dependence on maturation delay and death rate, J. Math. Biol., 49 (2004), pp. 188-200.

[14] J. K. Hale And S. M. V. Lunel, Introduction to Functional Differential Equations, SpringerVerlag, New York, 1993.

[15] M. P. Hassell and S. W. Pacala, Heterogeneity and the dynamics of host-parasitoid interactions, Philos. Trans. R. Soc. Lond. Ser. B, 330 (1990), pp. 203-220.

[16] A. Hastings, Age-dependent predation is not a simple process. I. Continuous time models, Theoret. Pop. Biol., 23 (1983), pp. 347-362.

[17] A. Hastings, Delays in recruitment at different trophic levels: Effects on stability, J. Math. Biol., 21 (1984), pp. 35-44.

[18] K. Engelborghs, T. Luzyanina, and D. Roose, Numerical bifurcation analysis of delay differential equations using DDE-BIFTOOL, ACM Trans. Math. Software, 28 (2002), pp. $1-21$.

[19] Y. Kuang, Delay Differential Equations, Academic Press, Boston, 1993.

[20] S. Leela, and V. Lakshmikantham, Differential and Integral Inequalities, Academic Press, New York, 1969.

[21] W. W. Murdoch, C. J. Briggs, And R. M. Nisbet, Dynamical effects of host size- and parasitoid state-dependent attacks by parasitoids, J. Animal Ecol., 66 (1997), pp. 542-556.

[22] W. W. Murdoch, R. M. Nisbet, S. P. Blythe, W. S. C. Gurney, and J. D. Reeve, An invulnerable age class and stability in delay-differential parasitoid-host models, Amer. Naturalist, 129 (1987), pp. 263-282.

[23] J. D. Murray, Mathematical Biology, Springer-Verlag, Berlin, 1989.

[24] R. M. Nisbet and W. S. C. Gurney, Modelling Fluctuating Populations, John Wiley \& Sons, New York, 1982.

[25] L. Nunney, The effect of long time delays in predator-prey systems, Theoret. Pop. Biol., 27 (1985a), pp. 202-221.

[26] L. Nunney, Absolute stability in predator-prey models, Theoret. Pop. Biol., 28 (1985b), pp. 209-232.

[27] L. Nunney, Short time delays in population models: A role in enhancing stability, Ecology, 66 (1985c), pp. 1849-1858.

[28] P. Turchin, A. D. Taylor, And J. D. Reeve, Dynamical role of predators in population cycles of a forest insect: An experimental test, Science, 285 (1999), pp. 1068-1071. 